\title{
Origin of barite deposits in dolomite-limestone units, Gazipasa, Eastern of Antalya: geology, geochemistry, statistics, sulfur isotope composition
}

\author{
Gulsum B. Kursun ${ }^{1}\left({ }^{\circ}\right.$, Mustafa G. Yalcin $^{1 * \otimes(1)}$ \\ ${ }^{1}$ University of Akdeniz, Antalya, 07058, Turkey \\ *Corresponding author: e-mail gurhanyalcin@akdeniz.edu.tr, tel. +0902423196381
}

\begin{abstract}
Purpose. Gazipasa region is one of the most important barite mineralization of Turkey. To investigate the main origin properties of Gazipasa barite deposits, conditions of their genesis and occurrence.

Methods. To investigate the basic geological, geochemical, mineralogical, statistical, sulfur isotopic properties of Gazipasa barite deposits, conditions of their genesis and occurrence.

Findings. Paragenesis of barites deposits can be presented as barite, galena sphalerite, pyrite, limonite, quartz and calcite. Also, there are lots of barite-galena ores around Gazipasa. In the wall rocks of vein, while barium occurence is low, limonite and galenite density is high. According to isotope analysis results, ${ }^{34} \mathrm{~S}$ ranks between 20.3 and 22.4 . As indicated in the Rare Earth Elements (REE) diagram, calculated values show that barites reflect sedimentary environment conditions. Barite formation in these reserves contains approximately $86-99 \% \mathrm{BaSO}_{4}$ and it was determined to be mostly found in dolomites and limestones as lode, vein and veinlet.
\end{abstract}

Originality. According to geological and mineralogical studies, barite formation in dolomite-limestone units occurred in sin-sedimentary stage conditions.

Practical implications. Barites in the region are used because of their high tenor and closeness to the harbor.

Keywords: barite, isotopic composition, sedimentary characteristics, ${ }^{34} \mathrm{~S}$, genesis

\section{Introduction}

The generation of barite deposits has been the center of attraction of geological, mineralogical, geochemical, genesis and isotope studies for many years [1]-[12].

There are many large and small dimensions of barite and galenite in a large area of the study region. In the Ziegler's research [13], he made geological observations, however he didn't mention about genesis of mines in the study area. Later, researchers like Striebel [14], Copuroglu [15], Gokce and Bozkaya [16], Sadiklar [17], Ayhan [18] and Bozkaya and Gokce [19] made investigations about barite in the same region and mentioned about geological, geochemical, petrographic and mineralogical features of research area. In the Barutoglu's research [20], it was explained that barites are hydrothermal-vein ore; however, Striebel [14] explained that, the sediment is form of barite deposit and upper Permian. In the Gokce and Bozkaya's researches [21], it was explained that barites came about first term of mineralization and it was coupling agent before galenite. It was stated that Galena and other minerals are observed together across the porous zones between the brecciated barite crystals and galenite are formed later (epigenetic) than barites and especially abun- dant in mylonitized regions. According to Gokce and Bozkaya [16], in X-ray diffraction (XRD) and microscopical investigations, it was stated that mines contain barite (80-85\%), galenite $(10-15 \%)$ and in small quantities of pyrite, sphalerite, quartz, limonite and calcite There are also $\mathrm{Ba}-\mathrm{Pb}$ ores in the Lower Permian-Triassic aged partially dolomitized limestones [22]. Briefly, there are different genesis comments about studies conducted in the region. Generally, in the issue of mineralization genesis, there is not proper association. So, for making genesis interpretation, there is a need to make isotope analysis and genesis interpretation of barites in the samples taken from barites in the region.

Aim of the study is the formation of the barites found in the Karalar district is in the form of factors affecting this formation. In this context, in the investigation area, it was made geological, geochemical and isotope studies with genesis interpretations.

\section{Materials and methods}

Study area takes in "Boyalik Mine" barite mine and "Buyuk Ocak" barite mine that take place Karalar district in Gazipasa subprovinve in Antalya province. Study area site location map is seen in the Figure 1. 


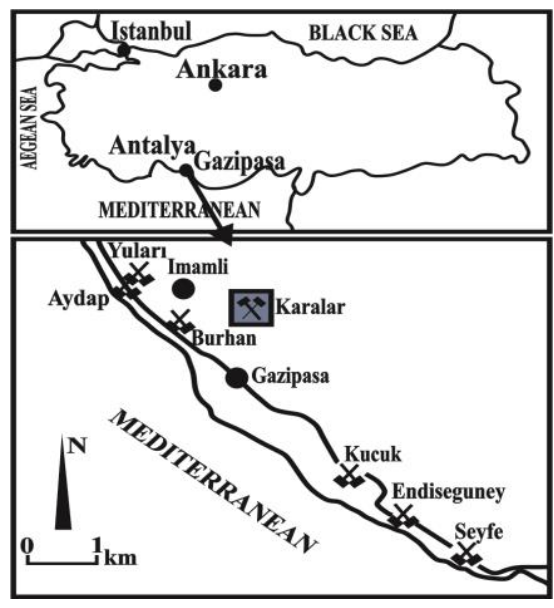

Figure 1. Location map of the study area

\subsection{Geology of study area}

Barite and galena deposits of Karalar distrinct are typical examples of barite-galena deposits that are common in Toros Mountain and contain carbonate [23]. In recent years, mining activities has concentrated on the area where "Buyuk Mine" and "Boyalik Mine" are located. Searches are generally about these mines and their environments. Toros mountains precipitated in the Cambrian-Tertiary time aged and it was stated that there were union reflecting different watershed characteristic [24]. These are named as Bolkar Mountain, Aladag, Geyik Mountain, Alanya, Bozkır and Antalya union [25]. Area of investigation takes place in Antalya union.

Antalya union also consists of different formations. These formations are named as Cakmak, Bickici and Camlica [26]. The Cakmak formation represents the oldest union and its base hasn't still formed. It wasn't certainly determined connections of Cile formation and Cakmak formation. It was just covered by Bickici formation that was given upper Permian age incompatibly. Limestone in the basement layer of Cakmak formation begins with shale alternation and continues to lift with micaceous shale (Fig. 2).

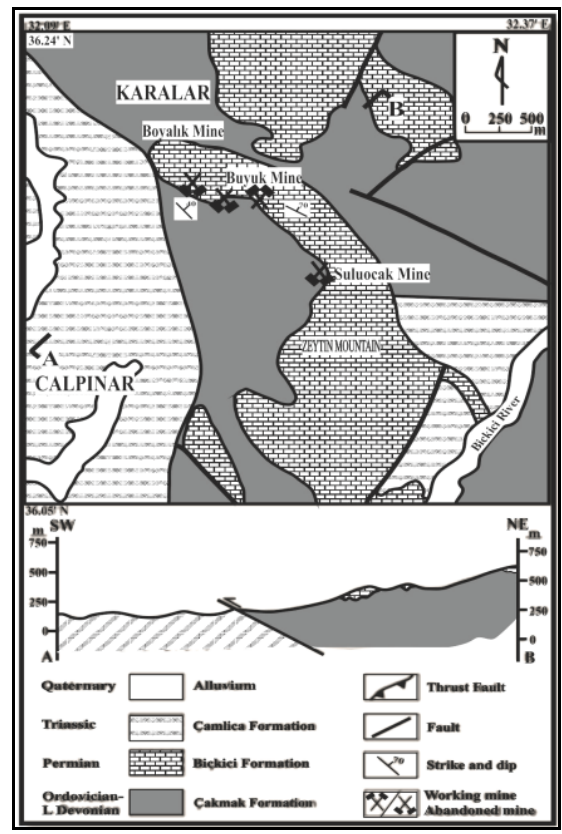

Figure 2. Local geological map of Karalar/Gazipasa (changed from [16], [26])
Corals like Bryozoa, Favosltos, Thamnapora fossil and trace elements like Cruziana Fucoides fossil were monitored within units. It was stated that these were Ordovician-Lower Devonian aged [27]. Mineralization is observed along the upper surface of the limestone blocks within the Bickici formation, in the form of lode in the ore-filled breccias and fault zones. This lode contains barite (80-85\%), galena (10-15\%), in small quantities of pyrite, sphalerite, quartz, limonit and calcite as major or minor) mineral. Macroscopic and microscopic features of sedimentaries show that barite evolved in the early mineralization period and it became mylonite before galenite crystallization [27]. Bickici formation begins with alternating of shale-crystalline limestone, micaceous siltstone, and sandy limestone time to time and goes on towards the upper layers to medium or thick bedded, calcite veins, gray colored and abundant fossiliferous limestones. Upper layer consists of stromatolite and oolitic sandy limestone. It is angularly incompatible with the Cile and Cakmak formations beneath the Bickici formation. Formation is determined as upper Permian [26].

Camlica formation begins with alternating of yellow argillaceous limestone and marl, goes on alternation of argillite, sandstone, sandstone with carbonate, siltstone towards the upper layers. At the top is the limestone containing Halobia, Daonella and radiolarite and in the flysch facies, there is sandstone, transitional to shale alternation. Formation is determined as middle-upper Triassic [26]. Camlica formation, turbiditic clastic (shale, mudstone and sandstone) and carbonate rocks (limestone, dolomite) were affected from shallow or pelagic in marine environments and late diagenetic [27]. Block of Cakmak and Bickici formations settled in Camlica formation in the Triassic period [24], [28], [29]. These formations are shown in Figure 2.

\subsection{Field studies and sampling}

Field studying concentrated on areas of barite mine and surrounding areas. The samples between BT1/BT17 was taken from "Boyalik Mine" and BT18/BT30 were taken from "Buyuk Ocak" barite gallery and its surroundings (Fig. 3).

In some parts of the "Buyuk Ocak" gallery, water spills and karst were observed on the roof, and barite, limonite, galena, calcite and azurite were observed in the heel.

Also, the main rocks of barite mineralization in the region were determined as dolomite and limestone. There are dark and light limestones and various levels of dolomitic limestones with barite in the area. Galenites were found in the fractures and cracks within the barites where the barite was milonitized by tectonic movements.

Samples taken from the land were sent to chemical analysis after necessary processing. At the end of the analysis, the values were transferred to the table in ppm or \%. X-ray diffractograms were used and Bruker's PDF-4 and SearchMatch software were used for XRD analyzes. X-ray powder diffraction data of the samples were refined with the Rietveld program Topaz 4.2 (Bruker AXS).

Off-line results in ${ }^{34} \mathrm{~S}$ isotope analysis were provided by $20 \%$ standard analysis. This assurance and control program allows the routine monitoring of both the measurements and the quality of the devices. All results are given in calibrated by certified reference materials (VCTD) reported in standard per mile (\%) and in accordance with international standards. Accuracy is based on repeated sample analysis in per mile $(\%)$. Based on primary or secondary standard analyzes, the results in the per-mile (\%) were transferred to the table. 
(a)

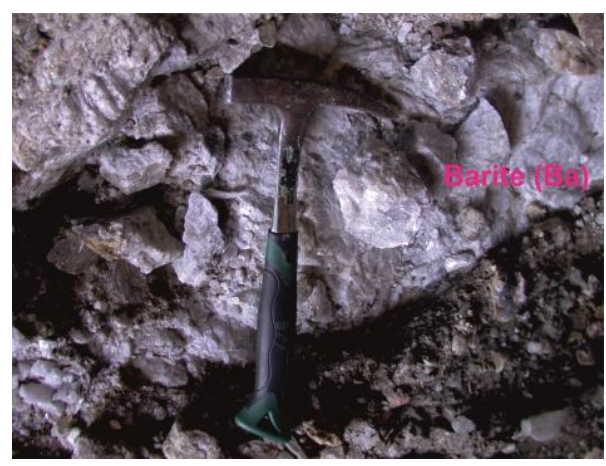

(b)

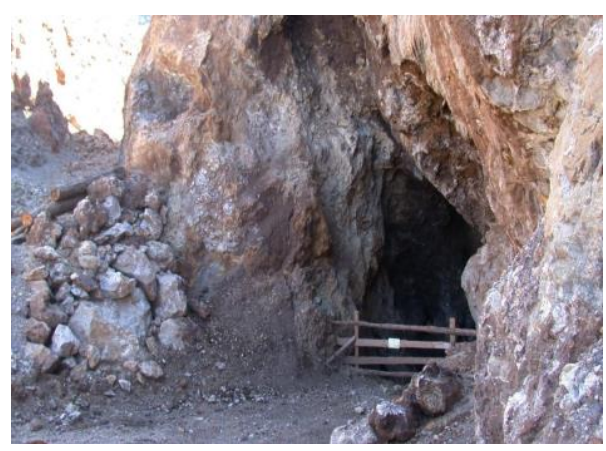

Figure 3. The barite in the "Boyalik Mine" gallery

Samples for sulfur were placed into tin capsules by scaling and the sulfur isotopic composition was measured using the MAT 253 Stable Isotope Ratio Mass Spectrometer attached to the Costech ECS 4010 Elemental Analyzer. ${ }^{34} \mathrm{~S}$ values, ${ }^{34} \mathrm{~S} /{ }^{\beta 2} \mathrm{~S}$ ratios in the sample, calculated by normalizing according to international standard of Vienna Canyon Diablo Troilite $(\mathrm{VCDT})$ and values, delta $(\delta)$ representations in per mil $(\%)$ units are reported using repeatable per mile (\%o) up to 0.2 .

\section{Results}

\subsection{X-ray diffraction (XRD)}

X-Ray diffraction of BT1/BT2/BT22/BT23/BT24 numbered samples was shown in Table 1 with detailed.

\section{Table 1. XRD diffraction results}

\begin{tabular}{lcccccc}
\hline \multirow{2}{*}{ Mineral } & $\begin{array}{c}\text { Chemical } \\
\text { formula }\end{array}$ & $\begin{array}{c}\mathrm{BT} 1, \\
\%\end{array}$ & $\begin{array}{c}\mathrm{BT} 2, \\
\%\end{array}$ & $\begin{array}{c}\mathrm{BT22} \\
\%\end{array}$ & $\begin{array}{c}\mathrm{BT23} \\
\%\end{array}$ & $\begin{array}{c}\mathrm{BT24} \\
\%\end{array}$ \\
\hline Barite & $\mathrm{BaSO}_{4}$ & 98.7 & 4.0 & 100 & 98.1 & 98.9 \\
Calcite & $\mathrm{CaCO}_{3}$ & 1.3 & 94.7 & - & - & - \\
Dolomite, & $\begin{array}{c}\mathrm{CaMg}\left(\mathrm{CO}_{3}\right)_{2} / \\
\mathrm{Ca}\left(\mathrm{Fe}^{2+}, \mathrm{Mg},\right.\end{array}$ & - & 0.6 & - & - & - \\
Ankerite & $\mathrm{Mn})\left(\mathrm{CO}_{3}\right)_{2}$ & & & & & \\
Quartz & $\mathrm{SiO}_{2}$ & - & 0.7 & - & 1.0 & 1.1 \\
Galena & $\mathrm{PbS}$ & - & - & - & 0.9 & - \\
Total & & 100 & 100 & 100 & 100 & 100 \\
\hline
\end{tabular}

According to results, the purest sample is BT22 sample taken from "Buyuk Ocak" gallery and it was determined that this sample is barite $100 \%$. BT22 was taken from side rocks in other examples and contains calcite 94\%. Samples of BT22, BT23 and BT24 contain $\mathrm{BaSO}_{4}$ over $98 \%$ (Figs. 4-7).

Sample BT2 was taken from side rock and it is referred to as calcite. Barites as side rock was found with rocks like galenite, dolomite, and calcite.

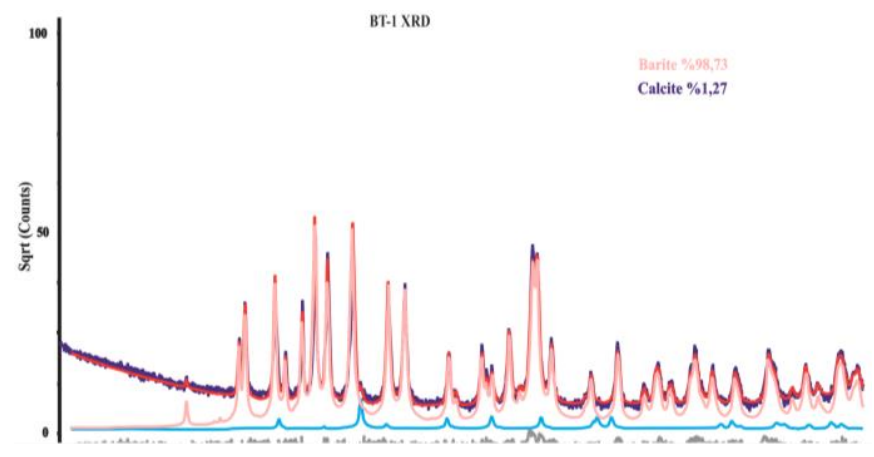

Figure 4. The view on the XRD diagram of the BT1 example

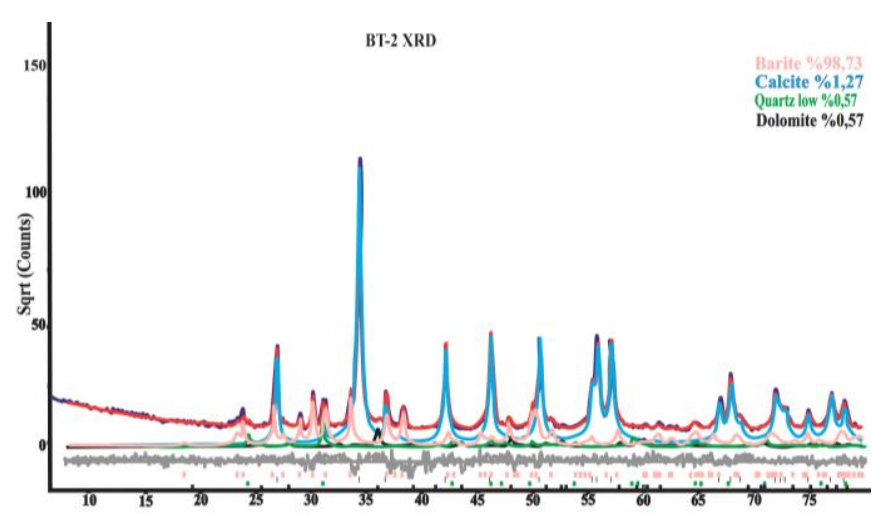

Figure 5. The view on the XRD diagram of the BT2 example

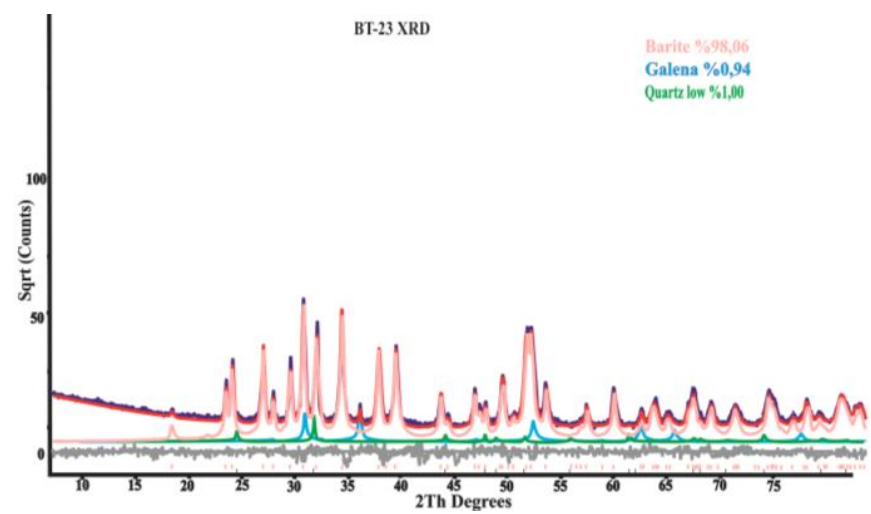

Figure 6. The view on the XRD diagram of the BT23 example

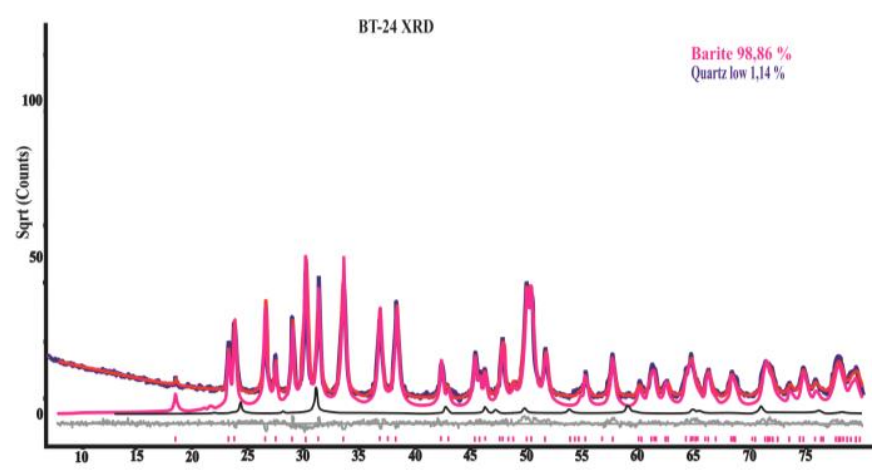

Figure 7. The view on the XRD diagram of the BT24 example

Calcite $94 \%$, barites $4 \%$, quartz $0.67 \%$ and dolomite $0.57 \%$ were found inside of BT2. Also, $98-100 \%$ barites were observed in other examples. 


\subsection{Geochemical analysis}

The table of XRF analysis results takes part in Tables 2 and 3. The REE and trace element contents tables (Tables 4 and 5) of the investigated area barite samples. Rare metal deposits, without any tectonic, magmatic and hydrothermal functions, indicate bearing types according to their relations with the rocks in which they are deposited. Since the rare earth element values of barite and wall samples are close to each other, other mean values are calculated and diagrams are drawn. Also, Tb, Gd, Dy, Er, Yb, La, Y, Ce were identified as heavy rare earth elements of Yitrium group in the samples.

Trace elements are usually less than $0.01 \%$ in rocks. It may also occur in concentrations other than normal. Sometimes these concentrations represent a mineral deposit and for example $\mathrm{Co}, \mathrm{Pb}, \mathrm{Cu}, \mathrm{Zn}$, etc. These elements can be identified and interpreted by adding them to the rock structure. In this context, XRF values have been examined and trace element table has been formed. Trace elements such as $\mathrm{Se}$ $\mathrm{As}-\mathrm{Zn}-\mathrm{Cu}-\mathrm{Mo}-\mathrm{Ni}$ were found in the samples.

Rare earth rates in barites have low $\mathrm{Ce} / \mathrm{Yb}$ values. High $\mathrm{Ce} / \mathrm{Yb}$ values show enrichment of light rare element in the samples, on the other hand low $\mathrm{Ce} / \mathrm{Yb}$ values show enrichment of high rare element. In the normalized rare earth element diagram (Fig. 8), these values showed positive zigzag anomalies for the relatively low remaining elements as positive peaks in La, Dy and Tm. Also, limestone and dolomite limestone in diagram exhibit positive $\mathrm{Eu}$ and negative $\mathrm{Ce}$ anomalies alike barites. These values are ranked at the maximum value range.

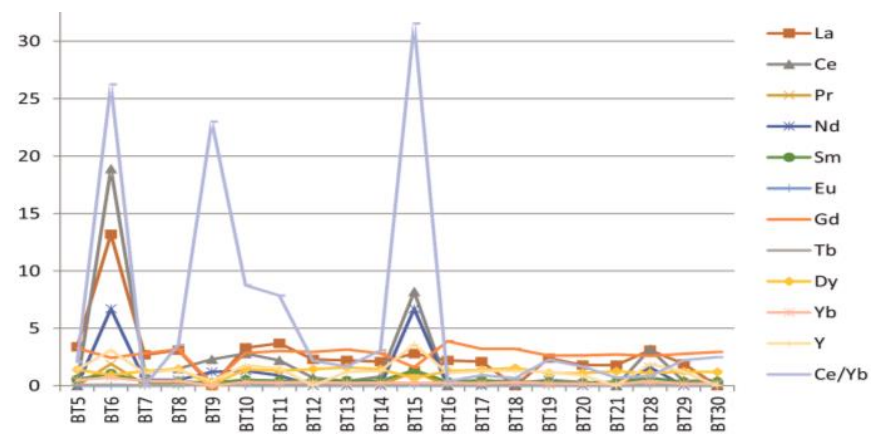

Figure 8. Diagram of REE

Table 2. Whole rock geochemistry

\begin{tabular}{|c|c|c|c|c|c|c|c|c|c|c|c|c|c|c|c|c|c|c|c|c|c|}
\hline Analyte & $\mathrm{SiO}_{2}$ & $\mathrm{Al}_{2} \mathrm{O}_{3}$ & $\mathrm{Fe}_{2} \mathrm{O}_{3}$ & $\mathrm{CaO}$ & $\mathrm{Na}_{2} \mathrm{O}$ & $\mathrm{MnO}$ & $\mathrm{Ba}$ & LOI & $\mathrm{SO}_{3}$ & $\mathrm{Sr}$ & TOT/C & TOT/S & $\mathrm{Ba}$ & $\mathrm{Ga}$ & $\mathrm{Hf}$ & $\mathrm{Sr}$ & $\mathrm{Ta}$ & $\mathrm{V}$ & $\mathrm{Zr}$ & $\mathrm{Y}$ & $\mathrm{La}$ \\
\hline Unit & $\%$ & $\%$ & $\%$ & $\%$ & $\%$ & $\%$ & $\%$ & $\%$ & $\%$ & $\%$ & $\%$ & $\%$ & PPM & PPM & PPM & PPM & PPM & PPM & PPM & PM & PPM \\
\hline MDL & 0.01 & 0.01 & 0.01 & 0.01 & 0.01 & 0.01 & 0.01 & -5.11 & .002 & .002 & 0.02 & 0.02 & 1 & 0.5 & 0.1 & 0.5 & 0.1 & 8 & 0.1 & 0.1 & 0.1 \\
\hline$\overline{\text { BT5 }}$ & 0.5 & .01 & 0.07 & 0.02 & 0.02 & .04 & 7.80 & -0.15 & 0.0 & 0.78 & $<0.02$ & 13.75 & 0000 & $<0.5$ & 1.9 & 308.5 & 6.8 & 21.0 & 0.3 & 1.4 & 3.4 \\
\hline BT6 & 30.70 & 1.11 & 3.99 & 6.32 & $<0.01$ & 0.09 & 29.12 & 5.61 & 10.0 & 0.40 & 1.40 & 7.50 & $>50000$ & $<0.5$ & 2.9 & 3214.8 & 3.6 & 23.0 & 0.3 & 3.1 & 13.2 \\
\hline BT7 & 0.65 & $<0.01$ & .01 & 0.37 & 0.03 & 0.04 & 58.06 & 0.15 & 0.0 & 0.63 & 0.08 & 14.00 & $>50000$ & 1.4 & 1.8 & 4774.6 & 6.2 & 11.0 & 68.2 & 1.1 & 2.7 \\
\hline BT8 & 1.00 & $<0.01$ & $<0.01$ & 1.23 & 0.02 & 0.04 & 56.72 & 0.68 & 10.0 & 0.63 & 0.24 & 13.48 & $>50000$ & 1.5 & 2.2 & 4739.9 & 4.4 & 14.0 & 0.7 & 1.4 & 3.1 \\
\hline ВT9 & 3.98 & 0.05 & 1.04 & 0.02 & $<0.01$ & $<0.01$ & 0374 & 7.02 & 10.0 & 0.02 & 0.26 & 11.40 & $>50000$ & 1.7 & $<0.1$ & 102.6 & 0.1 & $<8$ & 0.4 & 0.4 & 1.0 \\
\hline BT10 & 0.92 & $<0.01$ & $<0.01$ & 2.67 & 0.03 & 0.04 & 55.20 & 1.46 & 10.0 & 0.65 & 0.56 & 13.66 & $>50000$ & 1.1 & 1.7 & 4875.3 & 6.0 & $<8$ & 1.1 & 1.8 & 3.3 \\
\hline BT11 & 1.34 & 0.06 & 0.42 & 2.25 & $<0.01$ & 0.06 & 57.44 & 1.29 & $>10.0$ & 0.68 & 0.45 & 13.30 & $>50000$ & 1.2 & 1.8 & 4888.6 & 4.2 & $<8$ & 0.3 & 1.5 & 3.7 \\
\hline BT12 & $<0.01$ & $<0.01$ & 0.01 & 0.32 & 0.03 & 0.04 & 58.60 & 0.15 & 10.0 & 0.64 & 0.08 & 13.67 & $>50000$ & 0.9 & 1.7 & 4671.2 & 4.7 & $<8$ & 1.3 & 1.0 & 2.3 \\
\hline BT13 & $<0.01$ & $<0.01$ & $<0.01$ & 0.22 & 0.03 & 0.04 & 58.60 & 0.04 & 10.0 & 0.64 & 0.05 & 14.13 & $>50000$ & 0.9 & 1.7 & 4786.5 & 4.0 & $<8$ & 0.2 & 1.2 & 2.2 \\
\hline BT14 & 0.08 & 0.01 & $<0.01$ & 0.69 & 0.03 & 0.04 & 57.71 & 0.41 & 10.0 & 0.63 & 0.14 & 13.53 & $>50000$ & 0.9 & 2.1 & 4810.4 & 5.2 & $<8$ & 0.1 & 1.3 & 2.1 \\
\hline BT15 & $<0.01$ & $<0.01$ & 0.10 & 51.00 & $<0.01$ & 0.12 & 4.61 & 40.19 & 2.65 & 0.06 & 11.18 & 1.24 & 48312.00 & 1.0 & 0.4 & 849.2 & 0.9 & $<8$ & 0.2 & 3.6 & 2.8 \\
\hline BT16 & $<0.01$ & $<0.01$ & $<0.01$ & 0.18 & 0.03 & 0.04 & 58.78 & 0.07 & $>10.0$ & 0.61 & 0.04 & 13.96 & $>50000$ & 1.1 & 2.5 & 4577.5 & 6.0 & $<8$ & 0.3 & 1.1 & 2.2 \\
\hline BT17 & 0.05 & $<0.01$ & $<0.01$ & 0.20 & 0.02 & 0.04 & 57.89 & 0.15 & $>10.0$ & 0.61 & 0.04 & 13.96 & $>50000$ & 0.9 & 1.8 & 4652.3 & 4.7 & $<8$ & 0.2 & 1.3 & 2.1 \\
\hline BT18 & 1.59 & $<0.01$ & $<0.01$ & 0.21 & 0.02 & 0.04 & 57.62 & 0.17 & $>10.0$ & 0.71 & 0.04 & 13.58 & $>50000$ & 0.9 & 2.1 & 5374.5 & 5.5 & $<8$ & $<0.1$ & 1.3 & 2.0 \\
\hline BT19 & 1.63 & $<0.01$ & 0.09 & 0.98 & 0.02 & 0.05 & 56.72 & 0.80 & $>10.0$ & 0.61 & 0.19 & 13.52 & $>50000$ & 1.0 & 2.0 & 4511.1 & 4.7 & $<8$ & 0.6 & 1.2 & 2.4 \\
\hline BT20 & 0.64 & $<0.01$ & $<0.01$ & 0.10 & 0.03 & 0.04 & 58.42 & 0.07 & $>10.0$ & 0.63 & $<0.02$ & 14.07 & $>50000$ & 1.0 & 1.7 & 4674.5 & 5.5 & $<8$ & 0.2 & 0.9 & 1.8 \\
\hline BT21 & 0.41 & $<0.01$ & $<0.01$ & 0.08 & 0.03 & 0.04 & 58.15 & 0.06 & $>10.0$ & 0.64 & 0.02 & 13.97 & $>50000$ & 0.7 & 1.8 & 4691.5 & 3.4 & $<8$ & 0.1 & 1.0 & 1.8 \\
\hline BT28 & 8.88 & 0.17 & 0.50 & 6.47 & 0.04 & 0.09 & 48.48 & 4.91 & $>10.0$ & 0.54 & 1.42 & 11.25 & $>50000$ & 1.3 & 1.7 & 3977.1 & 2.8 & $<8$ & 3.0 & 1.9 & 3.1 \\
\hline BT29 & 1.54 & $<0.01$ & 0.04 & 0.45 & 0.04 & 0.04 & 57.17 & 0.35 & $>10.0$ & 0.60 & 0.09 & 13.53 & $>50000$ & 0.8 & 1.7 & 4511.4 & 4.9 & $<8$ & 0.3 & 1.2 & 1.9 \\
\hline ВT30 & 0.90 & 0.02 & 0.05 & 0.01 & 0.01 & 0.04 & 58.06 & 0.43 & $>10.0$ & 0.58 & 0.10 & 13.87 & $>50000$ & 0.7 & 2.0 & 4191.9 & 4.1 & $<8$ & 0.5 & 1.0 & 2.0 \\
\hline
\end{tabular}

Table 3. The continuation of Table 2

\begin{tabular}{|c|c|c|c|c|c|c|c|c|c|c|c|c|c|c|c|c|c|c|c|c|}
\hline Analyte & $\mathrm{Ce}$ & $\operatorname{Pr}$ & $\mathrm{Nd}$ & $\mathrm{Sm}$ & $\mathrm{Eu}$ & $\mathrm{Gd}$ & $\mathrm{Tb}$ & Dy & $\mathrm{Er}$ & $\mathrm{Yb}$ & $\mathrm{Cu}$ & $\mathrm{Pb}$ & $\mathrm{Zn}$ & $\mathrm{Ni}$ & As & $\mathrm{Cd}$ & $\mathrm{Sb}$ & $\mathrm{Ag}$ & $\mathrm{Au}$ & $\mathrm{Hg}$ \\
\hline Unit & PPM & PPM & PPM & PPM & PPM & PPM & PPM & PPM & PPM & PPM & PPM & PPM & PPM & PPM & PPM & PPM & PPM & PPM & PPM & PPM \\
\hline MDL & 0.1 & 0.02 & 0.3 & 0.05 & 0.02 & 0.05 & 0.01 & 0.05 & 0.03 & 0.05 & 0.1 & 0.1 & 1.0 & 0.1 & 0.5 & 0.1 & 0.1 & 0.1 & 0.5 & 0.01 \\
\hline BT5 & 1.0 & 0.05 & $<0.3$ & 0.56 & $*$ & 3.27 & 0.05 & 1.40 & $<0.03$ & 0.24 & 55.2 & $>100000$ & 14.0 & $<0.1$ & 9.9 & 0.5 & 12.7 & 4.2 & 2.4 & 1.69 \\
\hline BT6 & 18.9 & 1.90 & 6.7 & 1.07 & $*$ & 2.44 & 0.10 & 0.93 & 0.31 & 0.36 & 555.8 & 4553.1 & 185.0 & 5.5 & 308.4 & 0.5 & 48.2 & 0.4 & 1.7 & 3.98 \\
\hline BT7 & 1.2 & 0.12 & 0.5 & 0.34 & $*$ & 2.82 & 0.04 & 1.28 & $<0.03$ & 0.2 & 94.6 & 94.8 & 105.0 & 0.4 & 1.6 & 0.7 & 1.9 & 0.2 & 4.6 & 1.66 \\
\hline BT8 & 1.5 & 0.13 & 0.5 & 0.36 & $*$ & 3.18 & 0.06 & 1.48 & $<0.03$ & 0.21 & 67.1 & 209.3 & 39.0 & 0.2 & 1.7 & 0.7 & 1.6 & 0.1 & 0.8 & 1.78 \\
\hline ВT9 & 2.3 & 0.27 & 1.2 & 0.25 & 0.11 & 0.18 & 0.02 & 0.07 & $<0.03$ & $<0.05$ & 498.6 & $>100000$ & 1673.0 & 2.6 & 106.1 & 16.9 & 246.9 & 22.2 & $<0.5$ & 17.1 \\
\hline BT10 & 2.8 & 0.31 & 1.3 & 0.53 & $*$ & 2.86 & 0.08 & 1.43 & 0.07 & 0.16 & 47.2 & 2928.9 & 4.0 & $<0.1$ & 0.9 & $<0.1$ & 1.1 & 0.6 & $<0.5$ & 0.72 \\
\hline BT11 & 2.2 & 0.20 & 0.9 & 0.43 & $*$ & 3.03 & 0.06 & 1.321 & $<0.03$ & 0.14 & 124.7 & $>100000$ & 65.0 & $<0.1$ & 7.1 & 0.5 & 8.0 & 1.9 & $<0.5$ & 4.03 \\
\hline BT12 & 0.7 & $<0.02$ & $<0.3$ & 0.39 & $*$ & 2.94 & 0.05 & 1.43 & $<0.03$ & 0.16 & 10.2 & 53.2 & 8.0 & $<0.1$ & $<0.5$ & 0.4 & 0.5 & $<0.1$ & 2.4 & 0.23 \\
\hline BT13 & 0.4 & $<0.02$ & $<0.3$ & 0.45 & $*$ & 3.16 & 0.04 & 1.60 & $<0.03$ & 0.12 & 11.8 & 25.5 & 5.0 & $<0.1$ & $<0.5$ & 0.2 & 0.3 & $<0.1$ & 2.0 & 0.24 \\
\hline BT14 & 0.8 & 0.06 & $<0.3$ & 0.41 & $*$ & 2.82 & 0.05 & 1.44 & $<0.03$ & 0.13 & 8.7 & 337.9 & 5.0 & $<0.1$ & 0.7 & $<0.1$ & 0.9 & $<0.1$ & $<0.5$ & 0.26 \\
\hline BT15 & 8.2 & 1.24 & 6.7 & 1.36 & 1.16 & 1.62 & 0.12 & 0.66 & 0.18 & 0.13 & 4.3 & 35.3 & 3.0 & 1.7 & $<0.5$ & $<0.1$ & 0.2 & $<0.1$ & 3.0 & 0.1 \\
\hline BT16 & $<0.1$ & $<0.02$ & $<0.3$ & 0.34 & $*$ & 3.87 & 0.04 & 1.28 & $<0.03$ & 0.13 & 29.4 & 39.3 & 19.0 & 0.1 & 0.7 & 0.1 & 2.9 & $<0.1$ & $<0.5$ & 0.31 \\
\hline BT17 & 0.2 & $<0.02$ & $<0.3$ & 0.47 & $*$ & 3.21 & 0.05 & 1.39 & $<0.03$ & 0.11 & 25.8 & 32.2 & 16.0 & 0.1 & 0.6 & $<0.1$ & 2.0 & $<0.1$ & 0.6 & 0.3 \\
\hline BT18 & 0.2 & $<0.02$ & $<0.3$ & 0.35 & $*$ & 3.22 & 0.04 & 1.55 & $<0.03$ & 0.15 & 48.1 & 35.8 & 4.0 & $<0.1$ & 1.5 & $<0.1$ & 0.2 & $<0.1$ & 0.8 & 0.21 \\
\hline ВТ19 & 0.5 & 0.12 & 0.5 & 0.34 & $*$ & 2.62 & 0.05 & 1.12 & $<0.03$ & 0.11 & 55.1 & 121.2 & 14.0 & 0.4 & 12.8 & 0.2 & 0.7 & $<0.1$ & $<0.5$ & 2.49 \\
\hline ВТ20 & 0.3 & $<0.02$ & $<0.3$ & 0.28 & $*$ & 2.63 & 0.04 & 1.09 & $<0.03$ & 0.09 & 33.8 & 20.3 & 3.0 & $<0.1$ & 1.2 & $<0.1$ & $<0.1$ & $<0.1$ & $<0.5$ & 0.65 \\
\hline ВТ21 & $<0.1$ & $<0.02$ & $<0.3$ & 0.33 & $*$ & 2.72 & 0.04 & 1.25 & $<0.03$ & 0.07 & 32.2 & 24.2 & 3.0 & $<0.1$ & 1.2 & $<0.1$ & 0.1 & $<0.1$ & $<0.5$ & 0.47 \\
\hline ВТ28 & 3.2 & 0.37 & 1.6 & 0.69 & $*$ & 2.6 & 0.06 & 1.09 & 0.09 & 0.19 & 801.8 & 229.3 & 296.0 & 1.3 & 223.3 & 4.0 & 5.2 & 0.50 & 0.8 & 9.33 \\
\hline ВТ29 & 0.4 & $<0.02$ & $<0.3$ & 0.35 & $*$ & 2.79 & 0.04 & 1.23 & $<0.03$ & 0.09 & 43.0 & 62.0 & 9.0 & 0.4 & 8.4 & 0.1 & 0.6 & $<0.1$ & $<0.5$ & 1.82 \\
\hline ВТ30 & 0.5 & $<0.02$ & $<0.3$ & 0.34 & $*$ & 2.95 & 0.05 & 1.21 & $<0.03$ & 0.1 & 59.7 & 71.3 & 15.0 & 0.4 & 8.6 & $<0.1$ & 0.4 & $<0.1$ & $<0.5$ & 2.68 \\
\hline
\end{tabular}


Table 4. Trace element contents of barites

\begin{tabular}{|c|c|c|c|c|c|c|c|c|c|c|c|c|c|c|c|c|c|c|c|c|}
\hline Analyte & Dy & Ho & $\mathrm{Er}$ & $\mathrm{Tm}$ & $\mathrm{Yb}$ & $\mathrm{Lu}$ & Mo & $\mathrm{Cu}$ & $\mathrm{Pb}$ & $\mathrm{Zn}$ & $\mathrm{Ni}$ & As & $\mathrm{Cd}$ & $\mathrm{Sb}$ & $\mathrm{Bi}$ & $\mathrm{Ag}$ & $\mathrm{Au}$ & $\mathrm{Hg}$ & $\mathrm{Ti}$ & $\mathrm{Se}$ \\
\hline Unit & PPM & PPM & PPM & PPM & PPM & PPM & PPM & PPM & PPM & PPM & PPM & PPM & PPM & PPM & PPM & PPM & PPM & PPM & PPM & PPM \\
\hline MDL & 0.1 & 0.02 & 0.03 & 0.01 & 0.05 & 0.01 & 0.1 & 0.1 & 0.1 & 1.0 & 0.1 & 0.5 & 0.1 & 0.1 & 0.1 & 0.1 & 0.5 & 0.01 & 0.1 & 0.5 \\
\hline$\overline{\mathrm{BT} 5}$ & 1.40 & $<0.02$ & $<0.03$ & $<0.01$ & 0.24 & 0.02 & $<0.1$ & 55.2 & $>10000$ & 14.0 & $<0.1$ & 9.9 & 0.5 & 12.7 & $<0.1$ & 4.2 & 2.4 & 1.69 & 0.2 & $<0.5$ \\
\hline BT6 & 0.93 & 0.08 & 0.31 & 0.05 & 0.36 & 0.05 & 1.5 & 555.8 & 4553.1 & 185.0 & 5.5 & 308.4 & 0.5 & 48.2 & $<0.1$ & 0.4 & 1.7 & 3.98 & 21.0 & $<0.5$ \\
\hline BT7 & 1.28 & $<0.02$ & $<0.03$ & 0.01 & 0.2 & 0.02 & 0.6 & 94.6 & 94.8 & 105.0 & 0.4 & 1.6 & 0.7 & 1.9 & $<0.1$ & 0.2 & 4.6 & 1.66 & $<0.1$ & $<0.5$ \\
\hline BT8 & 1.48 & $<0.02$ & $<0.03$ & 0.02 & 0.21 & 0.02 & 0.2 & 67.1 & 209.3 & 39.0 & 0.2 & 1.7 & 0.7 & 1.6 & $<0.1$ & 0.1 & 0.8 & 1.78 & 0.1 & $<0.5$ \\
\hline ВT9 & 0.07 & $<0.02$ & $<0.03$ & $<0.01$ & $<0.05$ & $<0.01$ & 2.0 & 498.6 & $>10000$ & 1673.0 & 2.6 & 106.1 & 16.9 & 246.9 & 0.5 & 22.2 & $<0.5$ & 17.1 & 5.9 & 0.70 \\
\hline BT10 & 1.43 & 0.02 & 0.07 & 0.01 & 0.16 & 0.01 & $<0.1$ & 47.2 & 2928.9 & 4.0 & $<0.1$ & 0.9 & $<0.1$ & 1.1 & $<0.1$ & 0.6 & $<0.5$ & 0.72 & 0.1 & $<0.5$ \\
\hline BT11 & 1.31 & $<0.02$ & $<0.03$ & 0.02 & 0.14 & 0.01 & 0.2 & 124.7 & $>10000$ & 65.0 & $<0.1$ & 7.1 & 0.5 & 8.0 & $<0.1$ & 1.9 & $<0.5$ & 4.03 & 1.8 & $<0.5$ \\
\hline BT12 & 1.43 & $<0.02$ & $<0.03$ & 0.01 & 0.16 & 0.01 & $<0.1$ & 10.2 & 53.2 & 8.0 & $<0.1$ & $<0.5$ & 0.4 & 0.5 & $<0.1$ & $<0.1$ & 2.4 & 0.23 & $<0.1$ & $<0.5$ \\
\hline BT13 & 1.60 & $<0.02$ & $<0.03$ & $<0.01$ & 0.12 & $<0.01$ & $<0.1$ & 11.8 & 25.2 & 5.0 & $<0.1$ & $<0.5$ & 0.2 & 0.3 & $<0.1$ & $<0.1$ & 2.0 & 0.24 & $<0.1$ & $<0.5$ \\
\hline BT14 & 1.44 & $<0.02$ & $<0.03$ & 0.01 & 0.13 & 0.02 & $<0.1$ & 8.7 & 337.9 & 5.0 & $<0.1$ & 0.7 & $<0.1$ & 0.9 & $<0.1$ & $<0.1$ & $<0.5$ & 0.26 & $<0.1$ & $<0.5$ \\
\hline BT15 & 0.66 & 0.09 & 0.18 & 0.02 & 0.13 & $<0.01$ & $<0.1$ & 4.3 & 35.3 & 3.0 & 1.7 & $<0.5$ & $<0.1$ & 0.2 & $<0.1$ & $<0.1$ & 3.0 & 0.1 & $<0.1$ & $<0.5$ \\
\hline BT16 & 1.28 & $<0.02$ & $<0.03$ & $<0.01$ & 0.13 & $<0.02$ & $<0.1$ & 29.4 & 39.3 & 19.0 & 0.1 & 0.7 & 0.1 & 2.9 & $<0.1$ & $<0.1$ & $<0.5$ & 0.21 & $<0.1$ & $<0.5$ \\
\hline BT17 & 1.39 & $<0.02$ & $<0.03$ & $<0.01$ & 0.11 & $<0.01$ & $<0.1$ & 25.8 & 32.2 & 16.0 & 0.1 & 0.6 & $<0.1$ & 2.0 & $<0.1$ & $<0.1$ & 0.6 & 0.3 & $<0.1$ & $<0.5$ \\
\hline BT18 & 1.55 & $<0.02$ & $<0.03$ & $<0.01$ & 0.15 & $<0.01$ & $<0.1$ & 48.1 & 35.8 & 4.0 & $<0.1$ & 1.5 & $<0.1$ & 0.2 & $<0.1$ & $<0.1$ & 0.8 & 0.21 & $<0.1$ & $<0.5$ \\
\hline BT19 & 1.12 & $<0.02$ & $<0.03$ & $<0.01$ & 0.11 & $<0.01$ & 0.1 & 55.1 & 121.2 & 14.0 & 0.4 & 12.8 & 0.2 & 0.7 & $<0.1$ & $<0.1$ & $<0.5$ & 2.49 & 0.2 & $<0.5$ \\
\hline ВТ20 & 1.09 & $<0.02$ & $<0.03$ & $<0.01$ & 0.09 & $<0.01$ & $<0.1$ & 33.8 & 20.3 & 3.0 & $<0.1$ & 1.2 & $<0.1$ & $<0.1$ & $<0.1$ & $<0.1$ & $<0.5$ & 0.65 & $<0.1$ & $<0.5$ \\
\hline ВТ21 & 1.25 & $<0.02$ & $<0.03$ & $<0.01$ & 0.07 & $<0.01$ & $<0.1$ & 32.2 & 24.2 & 3.0 & $<0.1$ & 1.2 & $<0.1$ & 0.1 & $<0.1$ & $<0.1$ & $<0.5$ & 0.47 & $<0.1$ & $<0.5$ \\
\hline ВТ28 & 1.09 & 0.02 & 0.09 & 0.02 & 0.19 & 0.01 & 0.2 & 801.8 & 229.3 & 296.0 & 1.3 & 223.3 & 4.0 & 5.2 & $<0.1$ & 0.50 & 0.8 & 9.33 & 0.3 & $<0.5$ \\
\hline ВТ29 & 1.23 & $<0.02$ & $<0.03$ & $<0.01$ & 0.09 & $<0.01$ & 0.1 & 43.0 & 62.0 & 9.0 & 0.4 & 8.4 & 0.1 & 0.6 & $<0.1$ & $<0.1$ & $<0.5$ & 1.82 & $<0.1$ & $<0.5$ \\
\hline ВT30 & 1.21 & $<0.02$ & $<0.03$ & $<0.01$ & 0.1 & $<0.01$ & $<0.1$ & 59.7 & 71.3 & 15.0 & 0.4 & 8.6 & $<0.1$ & 0.4 & $<0.1$ & $<0.1$ & $<0.5$ & 2.68 & $<0.1$ & $<0.5$ \\
\hline
\end{tabular}

Table 5. REE contents of barites

\begin{tabular}{|c|c|c|c|c|c|c|c|c|c|c|c|c|}
\hline Analyte & $\mathrm{La}$ & $\mathrm{Ce}$ & $\operatorname{Pr}$ & $\mathrm{Nd}$ & $\mathrm{Sm}$ & $\mathrm{Eu}$ & $\mathrm{Gd}$ & $\mathrm{Tb}$ & Dy & $\mathrm{Yb}$ & $\mathrm{Y}$ & $\mathrm{Ce} / \mathrm{Yb}$ \\
\hline Unit & PPM & PPM & PPM & PPM & PPM & PPM & PPM & PPM & PPM & PPM & PPM & PPM \\
\hline BT5 & 3.4 & 1.0 & 0.05 & $<0.3$ & 0.56 & $*$ & 3.27 & 0.05 & 1.40 & 0.48 & 1.4 & 2.08 \\
\hline BT6 & 13.2 & 18.9 & 1.90 & 6.7 & 1.07 & $*$ & 2.44 & 0.1 & 0.93 & 0.72 & 3.1 & 26.25 \\
\hline BT7 & 2.7 & 1.2 & 0.12 & 0.5 & 0.34 & $*$ & 2.82 & 0.04 & 1.28 & 0.4 & 1.1 & 3.0 \\
\hline BT8 & 3.1 & 1.5 & 0.13 & 0.5 & 0.36 & $*$ & 3.18 & 0.06 & 1.48 & 0.42 & 1.4 & 3.57 \\
\hline BT9 & 1.0 & 2.3 & 0.27 & 1.2 & 0.25 & 0.11 & 0.18 & 0.02 & 0.07 & $<0.1$ & 0.4 & 23 \\
\hline BT10 & 3.3 & 2.8 & 0.31 & 1.3 & 0.53 & $*$ & 2.86 & 0.08 & 1.43 & 0.32 & 1.8 & 8.75 \\
\hline BT11 & 3.7 & 2.2 & 0.2 & 0.9 & 0.43 & $*$ & 3.03 & 0.06 & 1.31 & 0.28 & 1.5 & 7.86 \\
\hline BT12 & 2.3 & 0.7 & $<0.02$ & $<0.3$ & 0.39 & $*$ & 2.94 & 0.05 & 1.43 & 0.32 & 1.0 & 2.18 \\
\hline BT13 & 2.2 & 0. & $<0.02$ & $<0.3$ & 0.45 & $*$ & $3.16+$ & 0.04 & 1.6 & 0.24 & 1.2 & 1.67 \\
\hline BT14 & 2.1 & 0.8 & 0.06 & $<0.3$ & 0.41 & $*$ & 2.82 & 0.05 & 1.44 & 0.26 & 1.3 & 3.08 \\
\hline BT15 & 2.8 & 8.2 & 1.24 & 6.7 & 1.36 & 1.16 & 1.62 & 0.12 & 0.66 & 0.26 & 3.6 & 31.54 \\
\hline BT16 & 2.2 & $<0.1$ & $<0.02$ & $<0.3$ & 0.34 & $*$ & 3.87 & 0.04 & 1.28 & 0.26 & 1.1 & 0.38 \\
\hline BT17 & 2.1 & 0.2 & $<0.02$ & $<0.3$ & 0.47 & $*$ & 3.21 & 0.05 & 1.39 & 0.22 & 1.3 & 0.91 \\
\hline BT18 & 2.0 & 0.2 & $<0.02$ & $<0.3$ & 0.35 & $*$ & 3.22 & 0.04 & 1.55 & 0.3 & 1.3 & 0.67 \\
\hline BT19 & 2.4 & 0.5 & 0.12 & 0.5 & 0.34 & $*$ & 2.62 & 0.05 & 1.12 & 0.22 & 1.2 & 2.27 \\
\hline BT20 & 1.8 & 0.3 & $<0.02$ & $<0.3$ & 0.28 & $*$ & 2.63 & 0.04 & 1.09 & 0.18 & 0.9 & 1.67 \\
\hline BT21 & 1.8 & $<0.1$ & $<0.02$ & $<0.3$ & 0.33 & $*$ & 2.72 & 0.04 & 1.25 & 0.14 & 1.0 & 0.71 \\
\hline BT28 & 3.1 & 3.2 & 0.37 & 1.6 & 0.69 & $*$ & 2.6 & 0.06 & 1.09 & 0.38 & 1.9 & 0.84 \\
\hline ВТ29 & 1.9 & 0.4 & $<0.02$ & $<0.3$ & 0.35 & $*$ & 2.79 & 0.04 & 1.23 & 0.18 & 1.2 & 2.22 \\
\hline ВT30 & 2.0 & 0.5 & $<0.02$ & $<0.3$ & 0.34 & $*$ & 2.95 & 0.05 & 1.21 & 0.2 & 2.0 & 2.5 \\
\hline
\end{tabular}

According to data obtained from XRF analysis, diagrams of $\mathrm{Ce}_{N} / \mathrm{Yb}_{N}-\mathrm{Yb}_{N}-\mathrm{Ce}_{N} / \mathrm{Sm}_{N}-\mathrm{Ce}_{N} / \mathrm{Yb}_{N}$ were prepared (Table 6).

Table 6. $C e_{N} / \mathrm{Yb}_{N}-\mathrm{Yb}_{N}-\mathrm{Ce}_{N} / \mathrm{Sm}_{N}-\mathrm{Ce}_{N} / \mathrm{Yb}_{N}$ values

\begin{tabular}{lccccc}
\hline Sample & $\mathrm{Ce} / \mathrm{Yb}$ & $\mathrm{Yb}$ & $\mathrm{Sm}$ & $\mathrm{Ce} / \mathrm{Sm}$ & $\mathrm{Ce} / \mathrm{Yb}$ \\
\hline BT5 & 2.08 & 0.5 & 1.1 & 1.78 & 2.08 \\
BT6 & 26.25 & 0.7 & 2.1 & 17.67 & 26.25 \\
BT7 & 3.0 & 0.4 & 0.7 & 3.52 & 3.0 \\
BT8 & 3.57 & 0.4 & 0.7 & 4.17 & 3.57 \\
BT9 & 23 & $<0.1$ & 0.5 & 9.2 & 23 \\
BT10 & 8.75 & 0.3 & 1.1 & 5.28 & 8.75 \\
BT11 & 7.86 & 0.3 & 0.9 & 5.12 & 7.86 \\
BT12 & 2.18 & 0.3 & 0.8 & 1.79 & 2.18 \\
BT13 & 1.67 & 0.2 & 0.9 & 0.89 & 1.67 \\
BT14 & 3.08 & 0.3 & 0.8 & 1.95 & 3.08 \\
BT15 & 31.54 & 0.3 & 2.7 & 6.03 & 31.54 \\
BT16 & 0.38 & 0.3 & 0.7 & 0.28 & 0.38 \\
BT17 & 0.91 & 0.2 & 0.9 & 0.42 & 0.91 \\
BT18 & 0.67 & 0.3 & 0.7 & 0.57 & 0.67 \\
BT19 & 2.27 & 0.2 & 0.7 & 1.47 & 2.27 \\
BT20 & 1.67 & 0.2 & 0.6 & 1.07 & 1.67 \\
BT21 & 0.71 & 0.1 & 0.7 & 0.3 & 0.71 \\
BT28 & 0.84 & 0.4 & 1.4 & 4.64 & 0.84 \\
BT29 & 2.22 & 0.2 & 0.7 & 1.14 & 2.22 \\
BT30 & 2.5 & 0.2 & 2.4 & 1.47 & 2.5 \\
\hline
\end{tabular}

When these data were converted into chart, results are the most important datum showing that the barites in the study area come from the sediment. Diagrams of $\mathrm{Ce}_{N} / \mathrm{Yb}_{N}-\mathrm{Yb}_{N}-$ $\mathrm{Ce}_{N} / \mathrm{Sm}_{N}-\mathrm{Ce}_{N} / \mathrm{Yb}_{N}$ values are taking place Figures 9 and 10. The datum obtained, generated diagrams and barite values of graphics reflect ambient conditions. In this context, it was clearly observed that hydrothermal formation did not occur in the environment. As indicated in the diagram, calculated values show that barites reflect sedimentary environment conditions. According to XRF analysis results, when $\mathrm{Ba} / \mathrm{Sr}$ rates were calculated, values range from 350 to 500 and differ in 2 samples. These samples are determined as BT9 galenite and BT15 limonite and this can be shown in Table 7.

Factor analysis values of barite samples are as shown in Table 8. According to SPSS-Statistical Program, the first factor explains the high "Eigenvalues" of 19.917 and $35.567 \%$ of the total variance. The second factor represents eigenvalues of 14.628 and $26.121 \%$ of the total variance. The third factor represents eigenvalues of 8.835 and $15.777 \%$ of the total variance. The fourth factor represents eigenvalues of 4.020 and $7.179 \%$ of the total variance. The fifth factor represents eigenvalues of 2.569 and $4.587 \%$ of the total variance. 


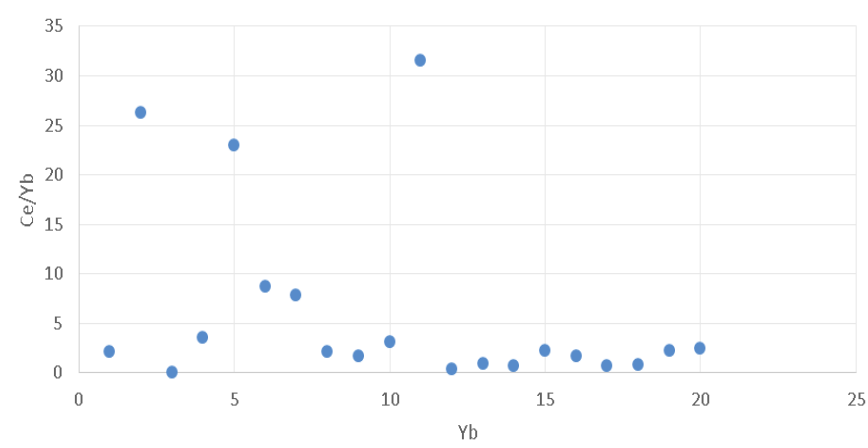

Figure 9. Normalized $C e_{N} / Y_{N}-Y_{N}$ diagram for barite samples taken from the study area

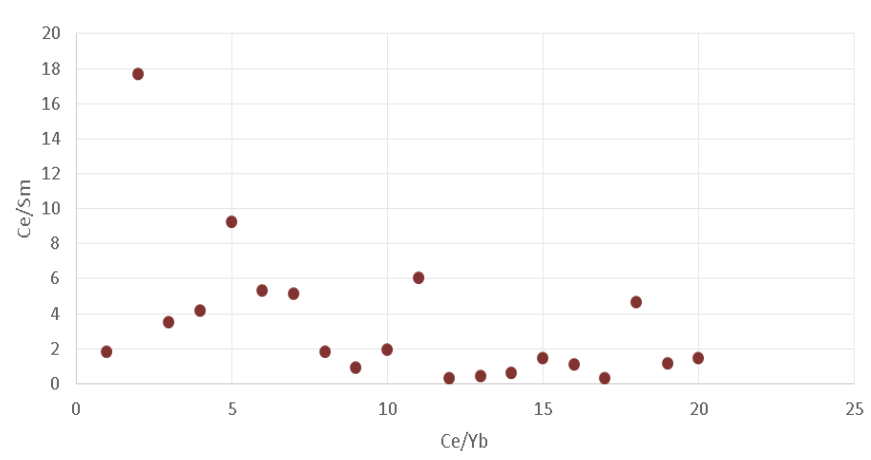

Figure 10. Normalized $C e_{N} / S_{N}-C e_{N} / Y_{b_{N}}$ diagram for barite samples taken from the study area

Table 7. According to XRF analysis results, Ba/Sr rates

\begin{tabular}{|c|c|c|c|c|c|}
\hline Sample & $\begin{array}{c}\text { XF700 } \\
\text { Ba } \\
\% \\
0.01\end{array}$ & XF700 & $\begin{array}{c}\text { XF700 } \\
\mathrm{Sr} \\
\% \\
0.002\end{array}$ & XF700 & $\begin{array}{c}\text { XF700 } \\
\mathrm{Ba} \text { (gr)/ } \\
\mathrm{Sr} \text { (gr) }\end{array}$ \\
\hline BT5 & 57.80 & 0.58 & 0.78 & 0.00156 & 370.04 \\
\hline BT6 & 29.12 & 0.29 & 0.40 & 0.00080 & 365.83 \\
\hline BT7 & 58.06 & 0.58 & 0.63 & 0.00127 & 458.61 \\
\hline BT8 & 56.72 & 0.57 & 0.63 & 0.00125 & 453.04 \\
\hline BT9 & 0.74 & 0.01 & 0.23 & 0.00046 & 16.09 \\
\hline BT10 & 55.20 & 0.55 & 0.65 & 0.0013 & 425.93 \\
\hline BT11 & 57.44 & 0.57 & 0.68 & 0.00135 & 424.85 \\
\hline BT12 & 58.60 & 0.59 & 0.64 & 0.00128 & 457.81 \\
\hline BT13 & 58.60 & 0.59 & 0.64 & 0.00128 & 459.25 \\
\hline BT14 & 57.71 & 0.58 & 0.63 & 0.00126 & 458.74 \\
\hline BT15 & 4.61 & 0.05 & 0.62 & 0.00124 & 37.18 \\
\hline BT16 & 58.78 & 0.59 & 0.61 & 0.00123 & 478.66 \\
\hline BT17 & 57.89 & 0.58 & 0.61 & 0.00121 & 478.43 \\
\hline BT18 & 57.62 & 0.58 & 0.71 & 0.00141 & 408.65 \\
\hline BT19 & 56.72 & 0.57 & 0.61 & 0.00122 & 464.92 \\
\hline BT20 & 58.42 & 0.58 & 0.63 & 0.00126 & 462.18 \\
\hline BT21 & 58.15 & 0.58 & 0.64 & 0.00127 & 456.44 \\
\hline BT28 & 48.48 & 0.48 & 0.54 & 0.00108 & 448.89 \\
\hline BT29 & 57.17 & 0.57 & 0.60 & 0.00119 & 479.61 \\
\hline BT30 & 58.06 & 0.58 & 0.58 & 0.00115 & 503.99 \\
\hline
\end{tabular}

Table 8. Factor analysis values of barite samples

\begin{tabular}{|c|c|c|c|c|c|c|}
\hline \multicolumn{7}{|c|}{ Total variance explanation rate } \\
\hline \multirow[b]{2}{*}{ Factor } & \multicolumn{3}{|c|}{ Initial eigenvalues } & \multicolumn{3}{|c|}{$\begin{array}{c}\text { Subtraction sum } \\
\text { of square estimates }\end{array}$} \\
\hline & Total & $\begin{array}{c}\text { Variance } \\
\text { explanation } \\
(\%)\end{array}$ & $\begin{array}{c}\text { Cumulative } \\
(\%)\end{array}$ & Total & $\begin{array}{c}\text { Variance } \\
\text { explanation } \\
(\%)\end{array}$ & $\begin{array}{c}\text { Cumulative } \\
(\%)\end{array}$ \\
\hline 1 & 19.917 & 35.567 & 35.567 & 19.917 & 35.567 & 35.567 \\
\hline 2 & 14.628 & 26.121 & 61.688 & 14.628 & 26.121 & 61.688 \\
\hline 3 & 8.835 & 15.777 & 77.465 & 8.835 & 15.777 & 77.465 \\
\hline 4 & 4.020 & 7.179 & 84.644 & 4.020 & 7.179 & 84.644 \\
\hline 5 & 2.569 & 4.587 & 89.231 & 2.569 & 4.587 & 89.231 \\
\hline
\end{tabular}

All elements are represented with 5 components across Karalar district. Taking into account the PCA analysis used five factors in Table 9, factor $\mathrm{F} 1$ contains $\mathrm{SiO}_{2}, \mathrm{MgO}, \mathrm{Na}_{2} \mathrm{O}$, $\mathrm{TiO}_{2}, \mathrm{Ba}, \mathrm{Sr}$, TOTS, Cs, Rb, Th, Zr, Ce, Dy, Ni, As, Tl; factor F2 contains $\mathrm{MnO}, \mathrm{Ba}, \mathrm{Yb}, \mathrm{Cd}, \mathrm{Sb}, \mathrm{Bi}, \mathrm{Ag}, \mathrm{Se}$; factor F3 contains $\mathrm{LOI}, \mathrm{SO}_{3}$, TOTC, $\mathrm{Ga}$; factor $\mathrm{F} 4$ contains $\mathrm{K}_{2} \mathrm{O}$, $\mathrm{Pr}, \mathrm{Nd}, \mathrm{Tm}, \mathrm{Mo}, \mathrm{Cu}$; factor $\mathrm{F} 5$ contains $\mathrm{P}_{2} \mathrm{O}_{5}, \mathrm{Tm}, \mathrm{Lu}, \mathrm{Pb}$.

Table 9. Correlation of component values of chemical elements

\begin{tabular}{|c|c|c|c|c|c|}
\hline \multicolumn{6}{|c|}{ Component } \\
\hline & 1 & 2 & 3 & 4 & 5 \\
\hline $\mathrm{SiO}_{2}$ & 0.787 & & & & \\
\hline $\mathrm{Al}_{2} \mathrm{O}_{3}$ & & 0.549 & & & \\
\hline $\mathrm{Fe}_{2} \mathrm{O}_{3}$ & 0.577 & & & & \\
\hline $\mathrm{MgO}$ & -0.74 & & & & \\
\hline $\mathrm{Na}_{2} \mathrm{O}$ & 0.809 & & & & \\
\hline $\mathrm{K}_{2} \mathrm{O}$ & & & & 0.627 & \\
\hline $\mathrm{MnO}$ & 0.506 & -0.83 & & & \\
\hline $\mathrm{TiO}_{2}$ & -0.72 & -0.56 & & & \\
\hline $\mathrm{P}_{2} \mathrm{O}_{5}$ & & & & & 0.564 \\
\hline $\mathrm{Sb}$ & 0.56 & -0.73 & & & \\
\hline $\mathrm{Bi}$ & & 0.846 & & & \\
\hline $\mathrm{Cr}_{2} \mathrm{O}_{3}$ & 0.581 & -0.63 & & & \\
\hline $\mathrm{Ba}$ & -0.85 & & & & \\
\hline LOI & 0.563 & & -0.81 & & \\
\hline $\mathrm{SO}_{3}$ & & & 0.9 & & \\
\hline $\mathrm{Sr}$ & -0.78 & & & & \\
\hline TOTC & & & -0.85 & & \\
\hline TOTS & -0.77 & & 0.609 & & \\
\hline Ba LF100 & & 0.848 & & & \\
\hline $\mathrm{Co}$ & 0.694 & & & & \\
\hline Cs & 0.72 & 0.557 & & & \\
\hline $\mathrm{Ga}$ & 0.526 & & & & \\
\hline Hf & & 0.607 & 0.7 & & \\
\hline $\mathrm{Nb}$ & 0.545 & & & & \\
\hline $\mathrm{Rb}$ & 0.741 & 0.53 & & & \\
\hline Sr LF100 & -0.76 & 0.526 & & & \\
\hline $\mathrm{Ta}$ & -0.67 & 0.497 & & & \\
\hline $\mathrm{Th}$ & -0.72 & -0.56 & & & \\
\hline $\mathrm{U}$ & & 0.696 & & & \\
\hline $\mathrm{Zr}$ & 0.731 & 0.548 & & & \\
\hline $\mathrm{Y}$ & 0.627 & 0.538 & -0.53 & & \\
\hline $\mathrm{La}$ & 0.677 & 0.651 & & & \\
\hline $\mathrm{Ce}$ & 0.879 & & & & \\
\hline $\operatorname{Pr}$ & & & & 0.652 & \\
\hline $\mathrm{Nd}$ & 0.534 & & & 0.523 & \\
\hline $\mathrm{Sm}$ & 0.699 & & -0.56 & & \\
\hline Gd & -0.67 & 0.655 & & & \\
\hline $\mathrm{Tb}$ & 0.56 & 0.525 & -0.56 & & \\
\hline $\mathrm{Se}$ & & 0.846 & & & \\
\hline Dy & -0.73 & 0.59 & & & \\
\hline Ho & -0.65 & & & & \\
\hline $\mathrm{Er}$ & -0.64 & & & & \\
\hline $\mathrm{Tm}$ & & & & 0.52 & -0.57 \\
\hline $\mathrm{Yb}$ & & -0.82 & & & \\
\hline $\mathrm{Lu}$ & & & & & -0.51 \\
\hline Mo & & & & 0.58 & \\
\hline \multicolumn{6}{|c|}{ Component } \\
\hline & 1 & 2 & 3 & 4 & 5 \\
\hline $\mathrm{Cu}$ & 0.673 & & & -0.53 & \\
\hline $\mathrm{Pb}$ & & & & & 0.599 \\
\hline $\mathrm{Ni}$ & 0.906 & & & & \\
\hline As & 0.792 & & & & \\
\hline $\mathrm{Cd}$ & & -0.84 & & & \\
\hline $\mathrm{Ag}$ & & -0.85 & & & \\
\hline $\mathrm{Tl}$ & 0.806 & & & & \\
\hline
\end{tabular}

BT16, 17, 8, 13, 3, 9, 15, 19, 12, 4, 10, 20, 14, 18, 2, 6 and 11 in the dendogram analysis of the Hierarchical Cluster Analysis which is based on the XRF results of 20 barite samples, among themselves; BT1 and 7 in between present similarities. BT1, 7, and 5 do not resemble the others of the previous examples. BT1, 5 and 7 are similar, but it was stated that BT5 is less similar among them. 
Similar groups in barite samples represent similarities among themselves of elements in Figure 11. However, samples of non-similarities have their own unique situation. According to the hierarchical group analysis dendogram made according to the barite specimens, it was observed that the diversity of the $Q$ type clump was generally in 2 (two) groups at the level of $50 \%$ similarity.

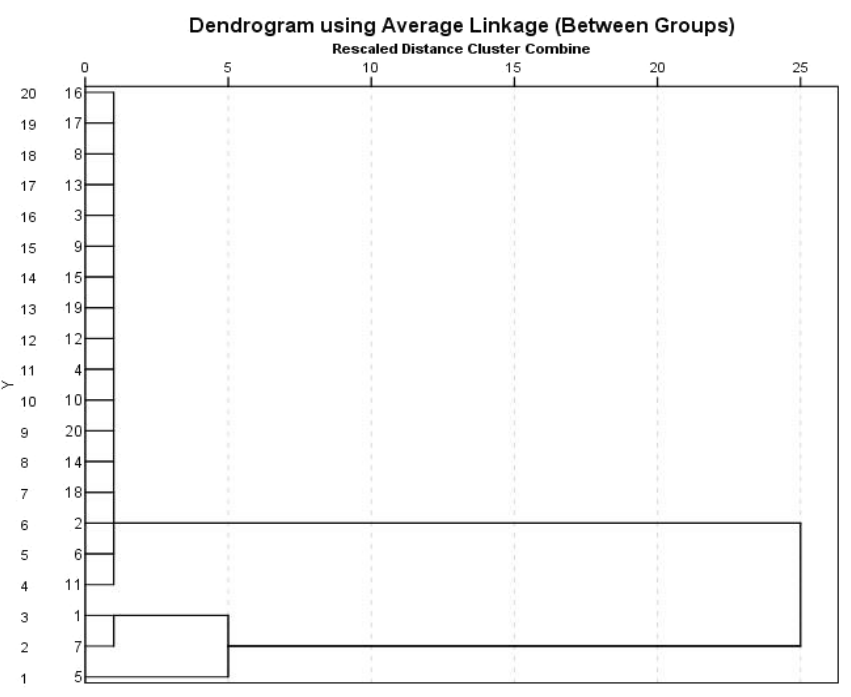

Figure 11. Hierarchical cluster analysis dendogram of barites

XRF results were calculated in Table 10, according to Summary and Anova. In calculation, datum of regression was looked at, according to Ba. Explanatories percentage of regression equation is $R^{2}=100 \%$ and there is no error margin. Analysis results give a very significant level of accuracy.

19 explanatory variables ( $\mathrm{Se}, \mathrm{Ce}, \mathrm{Pr}, \mathrm{Au}, \mathrm{SO}_{3}, \mathrm{Al}_{2} \mathrm{O}_{3}$, $\mathrm{P}_{2} \mathrm{O}_{5}, \mathrm{Ga}, \mathrm{Lu}, \mathrm{Mo}, \mathrm{Er}, \mathrm{Nb}, \mathrm{Tm}, \mathrm{K}_{2} \mathrm{O}, \mathrm{Hf}, \mathrm{Cu}, \mathrm{Pb}, \mathrm{Ni}$, TOTC) explain transformation of $\mathrm{Ba}$ element at a high level.

Table 10. According to model summary and anova of XRF data's

\begin{tabular}{ccccc}
\hline \multicolumn{5}{c}{ Model summary } \\
\hline Model & $R$ & $\begin{array}{c}\text { R } \\
\text { square }\end{array}$ & $\begin{array}{c}\text { Adjusted } R \\
\text { square }\end{array}$ & $\begin{array}{c}\text { Std. error of the } \\
\text { estimate }\end{array}$ \\
\hline 1 & $1.000^{\mathrm{a}}$ & 1 & . & . \\
\hline
\end{tabular}

Predictors: (constant), Se, Ce, $\mathrm{Pr}, \mathrm{Au}, \mathrm{SO}_{3}, \mathrm{Al}_{2} \mathrm{O}_{3}, \mathrm{P}_{2} \mathrm{O}_{5}, \mathrm{Ga}, \mathrm{Lu}, \mathrm{Mo}, \mathrm{Er}$, $\mathrm{Nb}, \mathrm{Tm}, \mathrm{K}_{2} \mathrm{O}, \mathrm{Hf}, \mathrm{Cu}, \mathrm{Pb}, \mathrm{Ni}$, TOTC

\begin{tabular}{ccccccc}
\hline \multicolumn{8}{c}{ Anova $^{\mathrm{a}}$} \\
\hline & Model & $\begin{array}{c}\text { Sum of } \\
\text { squares }\end{array}$ & df & $\begin{array}{c}\text { Mean } \\
\text { square }\end{array}$ & F & Sig. \\
\hline \multirow{2}{*}{1} & Regression & 5880.32 & 19 & 309.49 &. &. \\
& $\begin{array}{c}\text { Residual } \\
\text { Total }\end{array}$ & 0 & 0 &. & & \\
\hline
\end{tabular}

\footnotetext{
a. Dependent variable: $\mathrm{Ba}$

b. Predictors: (Constant), $\mathrm{Se}, \mathrm{Ce}, \mathrm{Pr}, \mathrm{Au}, \mathrm{SO}_{3}, \mathrm{Al}_{2} \mathrm{O}_{3}, \mathrm{P}_{2} \mathrm{O}_{5}, \mathrm{Ga}, \mathrm{Lu}, \mathrm{Mo}, \mathrm{Er}$, $\mathrm{Nb}, \mathrm{Tm}, \mathrm{K}_{2} \mathrm{O}, \mathrm{Hf}, \mathrm{Cu}, \mathrm{Pb}, \mathrm{Ni}$, TOTC
}

\subsection{Isotope analysis}

The results obtained for isotope analysis are as follows. QFIR protocols required for isotope analysis were made $10 \%$ coefficient of analysis for each of the following and the results aware shown in the range of QA and QC.

The value of ${ }^{32} \mathrm{~S} /{ }^{34} \mathrm{~S}$ in meteorites is 22.2 (Table 11), the value of ${ }^{32} \mathrm{~S} /{ }^{34} \mathrm{~S}$ in sulfur with sedimentary genesis is 22.44 ; if the ${ }^{32} \mathrm{~S} /{ }^{34} \mathrm{~S}$ value is 23 , the sulfuric mineral deposits are biogenic and if the ${ }^{32} \mathrm{~S} /{ }^{34} \mathrm{~S}$ ratio is around 22.2 it can be interpreted that it is of Ultrabasic and volcanic origin [30].
It shows that the origin of isotopic compositional sulfur content is magmatic, giving $\delta^{34} \mathrm{~S}$ values very close to each other and zeros [25].

Table 11. ${ }^{34} \mathrm{~S}$ isotope values measured from samples taken from barite mines of Karalar/Gazipasa

\begin{tabular}{lc}
\hline \multicolumn{1}{c}{ Example No. } & ${ }^{34} \mathrm{~S} \%$ ve VCDT \\
\hline BT27 & 22.4 \\
BT4 & 20.3 \\
BT3 & Inadequate S, \% \\
BT25 & 3.2 \\
BT26 & 9.0 \\
\hline
\end{tabular}

Being positive valence for marine origin and sulfur of negative value for bacterial and/or biological origin is thought [11]. In the studies at the Franklin Seamount in Western Woodlark Basin, the isotope analysis of silica flasks and barites in their contents were examined. Isotope analysis results of barites ( $\delta^{34} \mathrm{SV}-\mathrm{CDT}$ ) vary from 19.48 to 20.58 . It was stated that these values are clearer than typical diagenetic or cold leaking barites that frequently precipitate from $\mathrm{Ba}$ rich sediments in the sedimentary, but within the limits for typical hydrothermal barites. Based on the environmental conditions and the results of the analysis, it was determined that the barites found in the silica chimneys in the Franklin Seamount region of the West Woodlark Basin were of hydrothermal origin [11]. But no similarity was observed with the study area.

According to analyzes performed, the samples between BT1 and BT17 were taken from "Boyalik Mine" barite deposit and the genesis of the barites taken from here was evaluated on the basis of BT4. BT3 sample was taken from side rock and sufficient amount of sulfur was not detected. In the BT4 sample, $\delta^{34}$ S value was obtained as 20.3 . Based on analysis results and ambient conditions, it was thought to be the genesis of the barite deposit with sin-sedimentary genesis. Samples from BT18 and BT30 compiled from the study area represent Buyuk Ocak barite mine and BT27 sample was taken into consideration for making the origin interpretation from the samples sent to the isotope analyzes. $\delta^{34} \mathrm{~S}$ values in the BT25 and BT26 samples were determined as 3.2 and 9 and sulfur values were close to zero and BT25 and BT26 samples were found to contain side rocks that reduced the sulfur content with barite. In the BT27 sample, $\delta^{34} \mathrm{~S}$ value was 22.4 and it was determined to be of sin-sedimentary origin.

\section{Discussion}

For the formation of barite and galenite deposits ("Boyalik Mine" and "Buyuk Mine") which are observed intensively in the Central Anatolian Region especially in the Karalar distrinct [23] stated that within the limestones representing the Bickici Formation of the Permian age, there are vein type ores depending on fracture lines incompatible with layer planes [24]. Previous studies have shown that a large part of the galena- barites in the Gazipasa region have settled under Permian in sedimentary environment conditions. In addition, barites have always settled as a form of lenses that is compatible with the side rocks in certain stratigraphic levels, such as dolomitic, barite limestones and barite schists. Barites show structures like submarine slides [31]. The $\delta^{34} \mathrm{~S}$ isotope analyzes of Adana-Feke barite samples, was determined $\delta^{34} \mathrm{~S}$ values between 32.2 and 36.3\% [32]. It was determined by researchers that the barites present here are hydrothermal barite deposits. It is alternated with chart and 
carbonated rocks. Geochemical analyzes have also shown that there are no distinctive features of the hydrothermal universe in the basement and ceiling rocks [31]. In the deposits of study area, the lack of fragmental texture showing formation argued that the mineralization are of epigenetic genesis after the formation of limestones [31], [33], [34].

According to the study, it was not exactly determined that settling of $\mathrm{BaSO}_{4}$ in terms of chemical properties, in the first sedimentary rocks that have undergone late metamorphism in the sedimentary environment, initially only sedimentary formations or exhalative-sedimentary bed formations had occurred. In the studies conducted in Adana-Feke, in the diagrams prepared to values of $\mathrm{SrO}, \%$ while the Feke regional barites reflect hydrothermal barites, it is observed that the barrels of the Karalar region, representing our study area, are located at the sedimentary genesis.

According to [35], isotopic analysis of the barites between Sarkikaraagaç/Isparta and Huyuk/Konya revealed the ${ }^{34} \mathrm{~S}$ values as $\delta^{34} \mathrm{~S} 30.15 \%$ in their studies. When the isotope results are interpreted, the study area is compared with the sulfur isotope ratios of many barite deposits in the world and Barite mineralization in the region have been found to be similar to Mississippi valley type deposits [36]. It is believed that the barites in the study area represent hydrothermal barite deposits in the origin interpretation. When the isotope analysis studies performed in Karalar/Gazipasa region were compared with the results of the analysis in this region, it is thought that the isotope analysis values of the Karalar/Gazipasa region reflect sinsedimentary ambient conditions (Fig. 12).

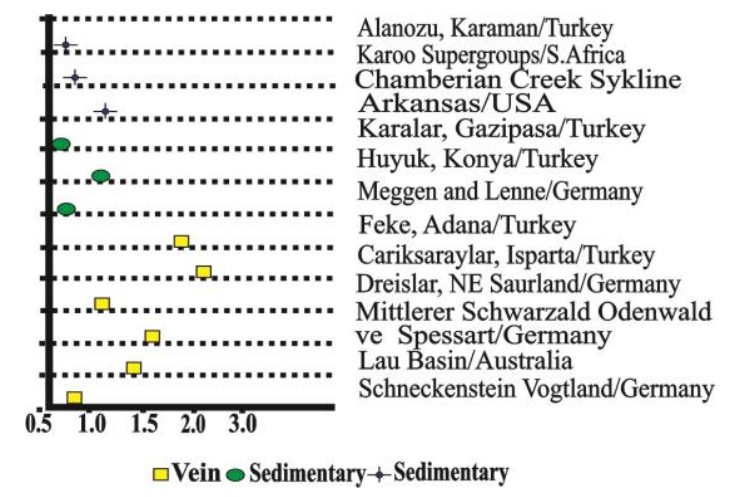

Figure 12. Barite types according to strontium oxide amount (taken from [14], [31], [32], [35])

Also, according to Cengiz and Kuscu's studies [35], they determined the $\mathrm{Ba}-\mathrm{Pb}-\mathrm{Zn}$ mineralization in the Permian aged crystallized limestone and dolomites in the Alanya massive in Karalar/Gazipasa region. The $\delta^{34} \mathrm{~S}$ isotope value measured in the galena and sphalerites in the $\mathrm{Pb}-\mathrm{Zn}$ deposits and occurrences of the Toros Mountains was measured and the isotope analysis value taken from the Karalar/Gazipasa region was determined as galence +4.6 and sphalerite +9.8 .

The $\mathrm{Pb}-\mathrm{Zn}$ deposits in the Central Toros Mountain were completely oxidized and the sulphates formed as a result of the decomposition were formed by reacting with the active side rock limestones and dolomites and forming carbonaceous ores and settling into the spaces formed as a result of carstification. It is determined that barites are found in deposits consisting mainly of sphalerite, galena, pyrite, marcasite, calcite, dolomite and quartz minerals [35].
According to Kuscu and Abdullah [37], isotopic analysis was performed in arsenic mineralization in Golbası (Isparta) region, and the barite samples of the mineralization were determined as having an $\delta^{34} \mathrm{~S}$ value of $030.32 \%$, ranging from 29.74 and $30.72 \%$.

In the area between Alanya and Gazipasa (Antalya) and Anamur (Mersin), it was determined that the barite was rich in sulphide barite and $\mathrm{Pb}-\mathrm{Zn}$ mineralization, galena-barite, galena - sphalerite - pyrite and barite - chalcopyrite in studies conducted by Cengiz and Ucurum [36]. Ba content of trace element contents of mineralizations; it was found to be at the lowest level of $29.15 \%$ and the highest at $50 \%$ in the barite mineralization. In this context, the relationship of mineralization with the side rocks, bearing shapes, mineral content, the order of formation of minerals and trace element content are thought to be the origin of mineralization consisting of epigenetic, medium-low temperature, side-rock interactive sea water and meteoric water mixing liquids [36].

In the previous studies, some researchers have defended that the genesis of these deposits are hydrothermal and metasomatic, while the other part, on the contrary, defended the formation of volcano sediments. Barutoglu's [20] report on the Karalar ore found in Gazipasa was published. According to Striebel [14], the ore revealed by the researcher as hydrothermal phylonian bed was interpreted as a sedimentary barite bed and formed in the Upper Permian. The geological observations of Ziegler [13] in his research do not contain any information on ores in the study area and its vicinity. In other researches, the textural structural features determined in the site where the ore mineralization was located suggested that barites did not coincide with galenites. Sadiklar and Amstutz [38], in pseudomatic folds, mentioned the occurrence of a migmatite at the beginning stage, but the similarity of quartz fillings with quartz veins and fracture fillings in other parts of the rock were found to be more effective in the presence of simple mobilizations during metamorphism. In this context, it was determined that firstly barites occurred and mylonitized with tectonic movements before formation of galenites [21]. In the clearances between barites, it was determined that fractures and cracks within the barites contain galenites and occur after barite mylonitization, is more frequent in parts where mylonitization is dense. In this context, research has revealed different opinions during previous studies in the field of study. In addition to these views, according to analyzes made in the barites taken from the study area, it is defended that area reflects sinsediment ambient conditions. The barites in the area were interpreted as genesis with the study and it was determined that there are not hydrothermal genesis.

\section{Conclusions}

As a result of XRD and XRF analyzes applied to the samples, the dominant mineral barite was determined as calcite, dolomite, galena, quartz. The contents of the main element of the samples taken from the study area are barium. With analyzing the geochemical samples taken from the barite and side stones in the region, variations and distributions of $\mathrm{Sr}$ and $\mathrm{Ba}$ values of the ore minerals were analyzed to determine the source and genesis of deposits. It has been stated that the amount of barite in the economic value limit is between 90.30 and $98.88 \%$ and the amount of the percentages decreases in inverse proportion to the increase in the amount of $\mathrm{SiO}_{2}$. Ba and $\mathrm{Sr}$ values of calcite and dolomites forming the side rocks 
of the deposits in the study area have not especially shown values reflecting a hydrothermal formation.

Limestones in the study area consist of KB-GD-trending, alternately ordered peaks. According to studies carried out in the field, various levels of limestone, in thin and dark gray thick layer and dolomitic limestones with barite were observed in the region. These differences are thought to be due to differences in age between rocks or differences in metamorphism processes. In the XRD diagrams of analyzes, it has been stated that $98 / 100 \%$ barite and calcite inside rocks (approximately 93\%) are main mineral. Also, minerals such as limonite, quartz, calcite, pyrite, galenite were found to join together at low levels.

In the previous studies in the study area, some of studies defended that deposits related to hydrothermal events are the genesis of such deposits while the others defended volcanosedimentary deposits. The ${ }^{34} \mathrm{~S}$ isotope ratios, deposit shapes, trace and mineral element contents of barites in the region are important factors for determining genesis of barites. In this context, according to isotope analyzes results, ${ }^{34} \mathrm{~S}$ value is between 20.3 and 22.4. It is thought that the genesis of the barites is sin-sedimentary in the results of the studies, observations and datum obtained.

In previous studies in the field, a part of the researchers have argued that the origin of such deposits is formed as hydrothermal metasomatic, while the other part is formed as volcano sedimentary. In other studies, the textural structural features determined at the site of mineralization in the area suggested that the barites did not occur simultaneously with the galenites. In this direction, it was found that the barites first formed and milonitized by tectonic movement before the formation of the galenites. It was determined that there were galenites in the fractures and cracks of barites and they were formed after the barite milonitization and they were found to be mostly in the parts where the mylonites were dense. In this context, the previous studies conducted in the field of study revealed different opinions. In addition to these views, it has been argued that the area reflects the sin-sedimentary environment conditions according to analyzes made in the barite taken from the study area. Barites in the field of study were interpreted originally and it was determined that they were not hydrothermal origin.

\section{Acknowledgements}

The study is supported with the FYL-2016-1832 Project by Akdeniz University Scientific Research Projects.

\section{References}

[1] Ehya, F. (2011). Rare earth element and stable isotope (O, S) geochemistry of barite from the Bijgan deposit, Markazi Province, Iran. Mineralogy and Petrology, 104(1-2), 81-93. https://doi.org/10.1007/s00710-011-0172-8

[2] Canet, C., Anadón, P., González-Partida, E., Alfonso, P., Rajabi, A., Pérez-Segura, E., \& Alba-Aldave, L.A. (2014). Paleozoic bedded barite deposits from Sonora (NW Mexico): Evidence for a hydrocarbon seep environment of formation. Ore Geology Reviews, (56), 292-300. https://doi.org/10.1016/j.oregeorev.2013.06.009

[3] Eickmann, B., Thorseth, I.H., Peters, M., Strauss, H., Bröcker, M., \& Pedersen, R.B. (2014). Barite in hydrothermal environments as a recorder of subseafloor processes: a multiple-isotope study from the Loki's Castle vent field. Geobiology, 12(4), 308-321. https://doi.org/10.1111/gbi.12086

[4] Asl, S.M., Jafari, M., Sahamiyeh, R.Z., \& Shahrokhi, V. (2015). Geology, geochemistry, sulfur isotope composition, and fluid inclusion data of
Farsesh barite deposit, Lorestan Province, Iran. Arabian Journal of Geosciences, 8(9), 7125-7139. https://doi.org/10.1007/s12517-014-1673-7

[5] Han, S., Hu, K., Cao, J., Pan, J., Xia, F., \& Wu, W. (2015). Origin of early Cambrian black-shale-hosted barite deposits in South China: Mineralogical and geochemical studies. Journal of Asian Earth Sciences, (106), 79-94. https://doi.org/10.1016/j.jseaes.2015.03.002

[6] Zhou, X., Chen, D., Dong, S., Zhang, Y., Guo, Z., Wei, H., \& Yu, H. (2015). Diagenetic barite deposits in the Yurtus Formation in Tarim Basin, NW China: Implications for barium and sulfur cycling in the earliest Cambrian. Precambrian Research, (263), 79-87. https://doi.org/10.1016/j.precamres.2015.03.006

[7] Magnall, J.M., Gleeson, S.A., Stern, R.A., Newton, R.J., Poulton, S.W., \& Paradis, S. (2016). Open system sulphate reduction in a diagenetic environment - Isotopic analysis of barite $(\delta 34 \mathrm{~S}$ and $\delta 180)$ and pyrite $(\delta 34 \mathrm{~S})$ from the Tom and Jason Late Devonian $\mathrm{Zn}-\mathrm{Pb}-\mathrm{Ba}$ deposits, Selwyn Basin, Canada. Geochimica et Cosmochimica Acta, (180), 146-163. https://doi.org/10.1016/j.gca.2016.02.015

[8] Jirásek, J., Dolníček, Z., Matýsek, D., \& Urubek, T. (2017). Genetic aspects of barite mineralization related to rocks of the teschenite association in the Silesian Unit, Outer Western Carpathians, Czech Republic. Geologica Carpathica, 68(2), 119-129. https://doi.org/10.1515/geoca-2017-0010

[9] Gao, J., Yang, R., Chen, J., Zheng, L., Cheng, W., \& Wei, H. (2017). Multiple proxies indicating methane seepage as the origin of Devonian large barite deposit in Zhenning-Ziyun, Guizhou, SW China. Ore Geology Reviews, (80), 18-26. https://doi.org/10.1016/j.oregeorev.2016.06.020

[10] Gornostayev, S.S., Crocket, J.H., Mochalov, A.G., \& Laajoki, K.V.O. (1999). The platinum-group minerals of the Baimka placer deposits, Aluchin horst, Russian Far East. Canadian Mineralogist, 37(5), 1117-1129.

[11] Ray, D., Banerjee, R., Balakrishnan, S., Paropkari, A.L., \& Mukhopadhyay, S. (2016). S- and Sr-isotopic compositions in barite-silica chimney from the Franklin Seamount, Woodlark Basin, Papua New Guinea: constraints on genesis and temporal variability of hydrothermal fluid. International Journal of Earth Sciences, 106(5), 1723-1733. https://doi.org/10.1007/s00531-016-1381-5

[12] Yang, X., Zhang, Z., \& Duan, S. (2017). Origin of the Mesoproterozoic Jingtieshan bedded barite deposit, North Qilian Mountains, NW China: Geochemical and isotope $(\mathrm{O}, \mathrm{S}, \mathrm{Sr})$ evidence. Geological Journal, (53), 21-32. https://doi.org/10.1002/gj.3088

[13] Ziegler, K.G.J. (1939). Mining and geological surveys in the Western Taurus region. 1st part. Af. T. A. Rap. No. 953 (unpublished).

[14] Striebel, H. (1965). Die Bleierz-Baryt-Lagerstaette von KaralarGazipasa (Turkei) und ihr geoiogischer Rahmen. PhD Thesis.

[15] Copuroglu, I. (1994). Mineralogical-petrographical studies and genesis of the Karalar (Gazipasa, Antalya) Galenite-Barite Mineralization. Maden Tetkik ve Arama Dergisi, 116(116), 1-13.

[16] Gokce, A., \& Bozkaya, G. (2007). Lead and sulphur isotopic studies of the barite-galena deposits in the Karalar area (Gazipasa - Antalya), Southern Turkey. Journal of Asian Earth Sciences, 30(1), 53-62. https://doi.org/10.1016/j.jseaes.2006.07.007

[17] Sadiklar, M.B. (1978). Schwerspat-und Bleiglanz-Vorkommen und ihre geologisch-petrogr. Lage im Gebiet der Dorfer K1c1k, E. Guney und Seyfe (Zeytinada) bei Gazipasa-Antalya (Turkei). Antalya, Turkey: Heidelberg.

[18] Ayhan, A. (1981). Pb-Zn deposits Aydap Yulari (Gazipasa-Antalya) and the origin of the problem remobilization. MTA, 95(96), 101-112.

[19] Bozkaya, G., \& Gokçe, A. (2004). Trace-and rare-earth element geochemistry of the Karalar (Gazipasa-Antalya) barite-galena deposits, southern Turkey. Turkish Journal of Earth Sciences, 13(1), 63-76.

[20] Barutoglu, O.H. (1942). Geological report of Garbi Taurus and Anamur Antalya. MTA Report (unpublished).

[21] Gökşe, A., \& Bozkaya, G. (2008). Fluid inclusion and stable isotope characteristics of the Karalar (Gazipaşa, Antalya) barite-galena deposits, Southern Turkey. Geology of Ore Deposits, 50(2), 145-154. https://doi.org/10.1134/s1075701508020050

[22] Kuscu, M., \& Cengiz, O. (2001). Karbonatlı kayaçlara bagli orta toroslar $\mathrm{Zn}-\mathrm{Pb}$ cevherlesmelerinin kukurt izotoplari incelemesi. Geological Bulletin of Turkey, 44(3), 59-73.

[23] Gokce, A., \& Bozkaya, G. (2003). Geology and fluid inclusion characteristics of the Karalar (Gazipaşa, Antalya) barite-galena deposits. Geol Bull Turk, (46), 1-16.

[24] Ozgul, N. (1984). Alanya tectonic window and geology of its western part. Ketin Symposium, (20), 21.

[25] Tas, A. (2009). Genesis investigation of Barite deposits in Eastern Toros mountain (Adana-Feke). Cukurova University Institute of Science and Technology Geological Engineering Departmente Doctorate Thesis.

[26] Ulu, U. (1983). Geological investigation in the Sugozu-Gazipasa, Antalya. Bulletin of Geological Engineering of Turkey, (16), 3-8. 
[27] Bozkaya, Ö., \& Yalçın, H. (2005). Diagenesis and very low-grade metamorphism of the Antalya unit: mineralogical evidence of Triassic rifting, Alanya-Gazipaşa, central Taurus belt, Turkey. Journal of Asian Earth Sciences, 25(1), 109-119. https://doi.org/10.1016/j.jseaes.2004.02.001

[28] Robertson, A.H.F., \& Woodcock, N.H. (1981). Gödene Zone, Antalya Complex: Volcanism and sedimentation along a Mesozoic continental margin, S.W. Turkey. Geologische Rundschau, 70(3), 1177-1214. https://doi.org/10.1007/bf01820188

[29] Robertson, A.H.F. (2000). Mesozoic-tertiary tectonic-sedimentary evolution of a south Tethyan oceanic basin and its margins in Southern Turkey. Geological Society, London, Special Publications, 173(1), 97-138. https://doi.org/10.1144/gsl.sp.2000.173.01.05

[30] Karakaya, M. (2009). Using of stable isotope in geology. Engineering Project Report. Ankara, Turkey.

[31] Ayan, M. (1979). Dunyada barit ve gelecegi. Jeoloji Muhendisligi Dergisi, 3(1), 47-64.

[32] Ozdogan, A.T., Uras, Y., \& Oner, F. (2017). Geochemistry of the barite deposits near Adana-Feke area (Eastern Taurides). Russian Geology and Geophysics, 58(11), 1349-1365. https://doi.org/10.1016/j.rgg.2017.11.003
[33] De Brodtkorb, M.K., Schalamuk, I.B.A., \& Ametrano, S. (1989). Barite and celestite Stratabound ore fields in Argentina. Nonmetalliferous Stratabound Ore Fields, 41-68. https://doi.org/10.1007/978-1-4684-6554-9_4

[34] Marumo, K. (1989). The barite ore fields of Kuroko-type of Japan Nonmetalliferous Stratabound Ore Fields, 201-231.

[35] Cengiz, O., \& Kuscu, M. (2002). Geochemical characteristics and origin of barite deposit between Sarkikaraagac (Isparta) and Huyuk (Konya). MTA Report, (123-124), 67-89.

[36] Cengiz, O., \& Ucurum, A. (2012). Geological-mineralogical characteristics and trace element contents of barite, sulfide- bearing barite and $\mathrm{Pb}-\mathrm{Zn}$ mineralizations (Antalya and Mersin Areas, Central TauridesTurkey). $65^{\text {th }}$ Geological Congress of Turkey Abstract Book, 408-411.

[37] Kuscu, M., \& Abdullah, S. (2017). Golbası (Isparta) damar tipi epitermal as cevherlesmesinin iz element ve kararli izotop jeokimyasi ozelliklerinin incelenmesi. Suleyman Demirel Universitesi Fen Bilimleri Enstitusu Dergisi, 21(3), 873-885.

[38] Sadiklar, M.B., \& Amstutz, G.C. (1981). Kıcık, Endiseguney and Seyfe (barite-galenite occurrences connected to the plate in the region Gazipasa/Antalya-Turkiye). MTA Journal, (95-96), 114-123.

\section{Походження баритових покладів у доломіто-вапнякових родовищах}

(Газіпаша, Східна Анталія): геологія, геохімія, статистика та ізотопний склад сірки

\section{Г.Б. Курсун, М.Г. Ялцін}

Мета. Вивчення основних геологічних, геохімічних, мінералогічних, статистичних, і сірчано-ізотопних властивостей баритових родовищ Газіпаша (Туреччина) з точки зору їх походження та умов формування.

Методика. Польові дослідження проводилися в районах баритових покладів Газіпаша. Зразки між ВТ1-ВТ17 були відібрані 3 шахти "Boyalik Mine”, a BT18-BT30 - зі штольні “Вuyuk Ocak”. Використовували рентгенівські дифрактограми, а для аналізу дифракції рентгенівських променів - програмне забезпечення Bruker PDF-4 i Search-Match. Дані порошкової рентгенографії зразків були уточнені за допомогою програми Ритвельда Topaz 4.2 (Bruker AXS). Автономні результати в ${ }^{34} \mathrm{~S}$ ізотопному аналізі отримані за допомогою $20 \%$ стандартного аналізу. Зразки для сірки поміщали в олов'яні капсули шляхом масштабування, ізотопний склад сірки вимірювали із використанням мас-спектрометра зі стабільним ізотопним співвідношенням MAT 253, прикріпленого до елементного аналізатору Costech ECS 4010.

Результати. Виявлено, що парагенезіс баритових родовищ представлений у вигляді бариту, галенового сфалериту, піриту, лімоніту, кварцу й кальциту. Причому в районі Газіпаша залягає також багато барито-галенових руд. Встановлено, що у бічних породах покладу спостерігається низький вміст барію, в той час як щільність лімоніту й галеніту вельми висока. Ізотопний аналіз показав, що вміст ізотопу сірки ${ }^{34} \mathrm{~S}$ коливається між 20.3 і 22.4. Аналіз діаграми рідкоземельних мінералів показує, що барити відображають екологічні умови їх відкладення. Частка баритів у цих родовищах становить близько 86-99\% у вигляді $\mathrm{BaSO}_{4}$, які в основному знаходяться в доломітах і вапняках у вигляді рудних тіл, жил і прожилків.

Наукова новизна. Доведено геологічними і мінералогічними дослідженнями, що утворення баритів у доломіто-вапнякових покладах відносяться до осадових порід.

Практична значимість. Проведені геохімічні та мінералогічні дослідження підтверджують високий вміст руди у баритовому родовищі Газіпаша. Завдяки цьому, а також близькості розташування до морського порту, руди є конкурентними і широко використовуються в різних галузях економіки на внутрішньому та зовнішньому ринках.

Ключові слова: барит, ізотопний склад, осадові характеристики, ізотоп ${ }^{34} \mathrm{~S}$, генезис

\section{Происхождение баритовых залежей в доломито-известняковых месторождениях (Газипаша, Восточная Анталия): геология, геохимия, статистика и изотопный состав серы}

\section{Г.Б. Курсун, М.Г. Ялцин}

Цель. Изучение основных геологических, геохимических, минералогических, статистических и серно-изотопных свойств баритовых месторождений Газипаши (Турция) с точки зрения их происхождения и условий формирования.

Методика. Полевые исследования проводились в районах баритовых залежей Газипаши. Образцы ВТ1-ВТ17 были отобраны из шахты “Boyalik Mine”, a BT18-BT30 - из штольни “Buyuk Ocak”. Использовали рентгеновские дифрактограммы, а для анализа дифракции рентгеновских лучей - программное обеспечение Bruker PDF-4 и Search-Match. Данные порошковой рентгенографии образцов были уточнены с помощью программы Ритвельда Topaz 4.2 (Bruker AXS). Автономные результаты в ${ }^{34}$ S изотопном анализе получены с помощью $20 \%$ стандартного анализа. Образцы для серы помещали в оловянные капсулы путем масштабирования, изотопный состав серы измеряли с использованием масс-спектрометра со стабильным изотопным соотношением MAT 253, прикрепленного к элементному анализатору Costech ECS 4010.

Результаты. Выявлено, что парагенезис баритовых месторождений представлен в виде барита, галенового сфалерита, пирита, лимонита, кварца и кальцита, причем в районе Газипаши залегает также много барито-галеновых руд. Установлено, что в боковых породах залежи наблюдается низкое содержание бария, в то время как плотность лимонита и галенита весьма высокая. Изотопный анализ показал, что содержание изотопа серы ${ }^{34} \mathrm{~S}$ колеблется между 20.3 и 22.4. Анализ диаграммы редкоземельных минералов показывает, что бариты отражают экологические условия их отложения. Доля баритов в этих месторождениях составляет около 86-99\% в виде $\mathrm{BaSO}_{4}$, которые в основном находятся в доломитах и известняках в виде рудных тел, жил и прожилок.

Научная новизна. Доказано геологическими и минералогическими исследованиями, что образования баритов в доломитоизвестняковых залежах относятся к осадочным породам.

Практическая значимость. Проведенные геохимические и минералогические исследования подтверждают высокое содержание руды, в баритовом месторождении Газипаши. Благодаря этому и близости к морскому порту, руды являются конкурентными и широко используются в различных отраслях экономики на внутреннем и внешнем рынках.

Ключевые слова: барит, изотопный состав, осадочные характеристики, изотоп ${ }^{34}$, генезис

\section{Article info}

Received: 01 October 2019

Accepted: 23 January 2020

Available online: 3 February 2020 